\title{
On the Rapid Weakening of Very Intense Tropical Cyclone Hellen (2014)
}

\author{
ADRIEN COLOMB AND TARIK KRIAT \\ Regional Specialized Meteorological Center for Tropical Cyclones La Réunion, Météo-France, Saint-Denis de La Réunion, France \\ MARIE-DOMINIQUE LEROUX \\ LACy, Laboratoire de l'Atmosphère et des Cyclones (UMR 8105/CNRS, Université de La Réunion, \\ Météo-France), Saint-Denis de La Réunion, France
}

(Manuscript received 24 August 2018, in final form 23 May 2019)

\begin{abstract}
In late March 2014, very intense Tropical Cyclone Hellen threatened the Comoros Archipelago and the Madagascan northwest coastline as it became one of the strongest tropical cyclones (TCs) ever observed over the Mozambique Channel. Its steep intensity changes were not well anticipated by operational forecasting models or by La Reunion regional specialized meteorological center forecasters. In particular, the recordsetting rapid weakening over the open ocean was not supported by usual large-scale predictors. AROME, a new nonhydrostatic finescale model, is able to closely reproduce these wide intensity changes. When benchmarked against available observations, the model is also consistent in terms of inner-core structure, environmental features, track, and intensity. In the simulation, a northwesterly 400-hPa environmental wind is associated with unsaturated air, while the classic $200-850$-hPa wind shear remains weak, and does not suggest a specifically unfavorable environment. The 400-hPa constraint affects the simulated storm through two pathways. Air with low equivalent potential temperature $\left(\theta_{e}\right)$ is flushed downward into the inflow layer in the upshear semicircle, triggering the decay of the storm. Then, direct erosion of the upper half of the warm core efficiently increases the surface pressure and also plays an instrumental role in the rapid weakening. When the storm gets closer to the Madagascan coastline, low $-\theta_{e}$ air can be directly advected within the inflow layer. Results illustrate on a real TC case the recently proposed paradigm for TC intensity modification under vertical wind shear and highlight the need for innovative tools to assess the impact of wind shear at all vertical levels.
\end{abstract}

\section{Introduction}

Over the last 20 years, significant progress has been made in the field of tropical cyclone (TC) numerical forecasting, mainly through a global reduction of track forecast errors. However, there is still room for improvement on the front of intensity guidance (DeMaria et al. 2014). In this context, intensity forecasts given by operational numerical weather prediction (NWP) models at the global scale, such as IFS $^{1}$ and GFS $^{2}$ are taken into account by TC forecasters, albeit with a limited

\footnotetext{
${ }^{1}$ Integrated Forecasting System, developed and run by the European Centre for Medium-Range Weather Forecasts (ECMWF).

${ }^{2}$ Global Forecast System, developed and run by the National Oceanic and Atmospheric Administration (NOAA).
}

Corresponding author: Adrien Colomb, adrien.colomb@meteo.fr confidence. Official Regional Specialized Meteorological Center (RSMC) intensity forecasts are mainly based on a subjective expertise of the influence of the synoptic environment on TCs and on models consensus, rather than on a single NWP model guidance. This subjective approach allowed the official forecasts to greatly outperform the automated guidance during the 1990s and early 2000s (DeMaria et al. 2014). Even though the NWP models have significantly improved their intensity forecast skill over the last decade, this subjective expertise is still used as one of the components in the elaboration of the official forecasts, especially in the warning time frame (24-48 $\mathrm{h}$ before landfall) where the model improvements have been the slowest (DeMaria et al. 2014). In an effort to streamline the subjective approach, statistical models have been developed based on the main environmental predictors for TC intensity, as documented in DeMaria and Kaplan (1994), Kaplan and DeMaria 
(2003), DeMaria et al. (2005) and Kaplan et al. (2010). These studies highlighted the influence of large-scale parameters, such as upper-level divergence, vertical wind shear (VWS) and sea surface temperature on TC intensity, and showed that these environmental factors can be used as predictors for TC intensity change. They particularly focused on rapid intensification (RI) cases for their potentially large intensity forecasts errors and disastrous consequences when occurring just before landfall. For instance, Kaplan et al. (2010) established that TCs located within a particularly conducive environment are much more likely to undergo RI even though the skill of statistical models remains limited for these extreme events.

However, the analysis of the large-scale predictors alone does not properly explain the observed intensity changes of some tropical systems. Inner-core processes, like vortex Rossby waves (Wang 2002; Montgomery and Kallenbach 1997; Corbosiero et al. 2012), vortical hot towers (Montgomery et al. 2006; Hendricks et al. 2004) and eyewall replacement cycles (Sitkowski et al. 2011), also play a major role in driving TC intensity changes.

In the southwestern Indian Ocean (SWIO) basin, very intense tropical cyclone (VITC) Hellen (2014) is one of those systems that did not seem to obey the classic environmental conditions and that challenged TC intensity prediction. VITC Hellen underwent unprecedented up and down intensity changes with a very rapid intensification ( $70 \mathrm{kt}$, or $36 \mathrm{~m} \mathrm{~s}^{-1}$, in $24 \mathrm{~h}$ ) followed by an even more drastic weakening ( $85 \mathrm{kt}$, or $44 \mathrm{~m} \mathrm{~s}^{-1}$, in $18 \mathrm{~h}$ ) before making landfall in Madagascar. This event highlighted a lack of skill in operational TC intensity guidance in the SWIO, as no NWP model was able to correctly forecast the magnitude nor the timing of the rapid intensification and rapid weakening. In particular, the processes leading to the rapid weakening of the VITC over the ocean remained "unclear," as stated in the official RSMC advisory issued at 0600 UTC 31 March 2014.

While RI processes have been under close scrutiny over the last decade, the fast decay of powerful and mature TCs has generally been drawing much less attention. Fast decaying systems are supposedly less threatening to infrastructure and human lives due to their decreasing strength, however, false alerts due to unpredicted rapid weakening before landfall result in costly and unnecessary massive evacuation of coastal populations and loss of credibility of the warning system. Moreover, understanding the processes involved in rapid weakening may be critical in pursuing the improvement of intensity guidance. The analysis of Hurricane Lili's (2002) rapid weakening is one study that discussed rapid weakening, and highlights the role of low-level dry air intrusion (Zhang et al. 2007). Since then, recent theoretical experiments using nonhydrostatic, finescale NWP models initialized with a TC-like vortex unraveled the thermodynamic impact of environmental VWS on TC intensity modification. Riemer et al. (2010) and more recently Onderlinde and Nolan (2017) highlighted the importance of low equivalent potential temperature $\left(\theta_{e}\right)$ air injections into the boundary layer that depress the near-core $\theta_{e}$ values, which subsequently weaken the updrafts within the eyewall. This mechanism is also combined to a ventilation of the midlevel warm core by the VWS (Tang and Emanuel 2010). This upsets the Carnot-cycle TC heat engine described by Emanuel (1986). The frustrated TC thermodynamic cycle is thus expected to yield a reduced TC intensity. A complete overview of the most recent publications on this subject is given in the introduction of Onderlinde and Nolan (2017). The thermodynamic approach addressing the effects of the advection of lowentropy air into the TC inner core is complementary to the dynamical approach (Tang and Emanuel 2010), the latter focusing on the kinematics of the interaction between the TC vortex and the environmental wind shear constraint (Frank and Ritchie 2001; Cram et al. 2007). Note that these studies are exclusively based on idealized experiments. There are few case studies confirming the significance of these processes for real TCs. Molinari et al. (2013) did focus on episodic outbreaks of convection affecting Tropical Storm Edouard (2014) and linked them to low $-\theta_{e}$ air intrusions observed in the boundary layer by U.S. Air Force reconnaissance flights. But this system was already rather weak during the period of interest. We focus here on the mechanisms that can downgrade a system from a VITC to a moderate tropical storm in just $18 \mathrm{~h}$ (the TC classification used in the SWIO basin is detailed in section 2).

The new nonhydrostatic, finescale model developed by Météo-France is able to realistically reproduce VITC Hellen's intensity, and gives an opportunity to investigate the very rapid weakening of Hellen over the open ocean. This study aims at (i) verifying that VITC Hellen is correctly represented in the numerical simulation, (ii) exploring the processes leading to its record-setting weakening and (iii) linking the model results to the recent theories presented above. The manuscript is organized as follows. Section 2 presents an overview of the storm and the model setup, and compares the simulated storm to available observations to validate its representativity. Results are presented in section 3 and are divided into five subsections. Section 3 a presents the environmental conditions at peak intensity, just before the onset of rapid weakening, and highlights the physical processes occurring at $400 \mathrm{hPa}$. Section $3 \mathrm{~b}$ investigates the processes at play in the low levels, namely the vertical injections of low- $\theta_{e}$ air in the inflow layer and the layer-wise advection from the Madagascan landmass. 
Section $3 c$ presents the vertical evolution of the upshear quadrant in the simulated storm. Section $3 d$ investigates the warm-core erosion and the advection of very dry air at $400 \mathrm{hPa}$. Section $3 \mathrm{e}$ uses the spindown theory to demonstrate the instrumental role of the upper-level warm-core erosion in the rapid weakening. Some additional points are discussed in section 4. Finally, the conclusion gives a summary of the results and draws recommendations in an operational context.

\section{Data and methods}

\section{a. Overview of very intense Tropical Cyclone Hellen (2014)}

The SWIO basin has a specific terminology and classification for tropical systems based on their 10-min average maximum wind speed. In the SWIO, the term "tropical cyclone" is dedicated to systems whose maximum winds (VMAX) reach or exceed $64 \mathrm{kt}$ (VMAX $\geq$ $32.9 \mathrm{~m} \mathrm{~s}^{-1}$ ) in at least a quadrant of the surface circulation. A system is assigned a name when it reaches the moderate tropical storm stage with winds reaching or exceeding $34 \mathrm{kt}$ (VMAX $\geq 17.4 \mathrm{~m} \mathrm{~s}^{-1}$ ). If a system intensifies further, it goes through the following stages: severe tropical storm (VMAX $\geq 48 \mathrm{kt}$ or $24.6 \mathrm{~m} \mathrm{~s}^{-1}$ ), tropical cyclone (VMAX $\geq 64 \mathrm{kt}$ or $32.9 \mathrm{~m} \mathrm{~s}^{-1}$ ), intense tropical cyclone (VMAX $\geq 90 \mathrm{kt}$ or $46.3 \mathrm{~m} \mathrm{~s}^{-1}$ ) and very intense tropical cyclone (VITC, VMAX $\geq 116 \mathrm{kt}$ or $59.6 \mathrm{~m} \mathrm{~s}^{-1}$ ).

VITC Hellen was the fourteenth system monitored by RSMC La Réunion in the SWIO during the 2013/14 cyclone season. The cyclogenesis took place in March 2014 in a very unusual location offshore Africa between northern Mozambique and the Comoros. At the beginning of the intensification phase, the VWS was weak and associated with relative humidities greater than $80 \%$ at all levels.

The system then tracked southeastward, a rather atypical track, thus threatening the Comoros archipelago and the Madagascan northwest seaboard (Fig. 1). VITC Hellen was also exceptional in regards to its extreme intensity changes. According to the RSMC best track (BT) data, mainly based on Dvorak estimates (Dvorak 1984), the system nearly gained $70 \mathrm{kt}^{3}\left(36 \mathrm{~m} \mathrm{~s}^{-1}\right)$ in $24 \mathrm{~h}$ between 29 and 30 March. This rate is more than twice the intensification rate for rapidly intensifying systems over the basin (Leroux et al. 2018, $30 \mathrm{kt}$ or $15.4 \mathrm{~m} \mathrm{~s}^{-1}$ ). At 1800 UTC 30 March, Hellen reached

\footnotetext{
${ }^{3} 10$-min average wind speed following the World Meteorological Organization standards.
}

its peak intensity estimated at $125 \mathrm{kt}\left(64 \mathrm{~m} \mathrm{~s}^{-1}\right)^{4}$, corresponding to a category 5 hurricane on the Saffir-Simpson hurricane wind scale. The storm then weakened at an even greater rate than it had intensified, losing $85 \mathrm{kt}$ $\left(44 \mathrm{~m} \mathrm{~s}^{-1}\right)$ in the following $18 \mathrm{~h}$ while remaining over open water (corresponding to an abnormal fall of 3.5 on the Dvorak intensity scale). Over the SWIO basin, the climatological threshold for rapid weakening has been assessed at $27 \mathrm{kt}\left(14 \mathrm{~m} \mathrm{~s}^{-1}\right)$ in $24 \mathrm{~h}$ over the $1999-2016$ period (Leroux et al. 2018). As a comparison, Hurricane Lili (2002) lost $40 \mathrm{kt}$ in $24 \mathrm{~h}$ and still ranks in the first percentile of the fastest 24-h decays in the North Atlantic basin (Frederick 2003). According to the National Hurricane Center's BT data, Hurricane Patricia (2015) also underwent a drastic oversea intensity loss, but it landed on the Mexican coastline while its rapid weakening phase was still ongoing. Patricia eventually lost $44 \mathrm{kt}$ $\left(23 \mathrm{~m} \mathrm{~s}^{-1}\right)$ in $17 \mathrm{~h}$ over the open ocean.

After losing most of its power over water, Hellen eventually landed on the Madagascan coastline as a moderate tropical storm around 1600 UTC 31 March, and only remained over land for the next $3 \mathrm{~h}$ before tracking back over sea as a remnant low.

In just two and a half days, Hellen went up and down the Dvorak scale, as TC forecasters chose to break the Dvorak constraints regarding intensity changes (normally restraining 24-h intensity changes to \pm 2.0 ) to keep up with the major changes observed in the TC structure. These rapid intensity changes were probably favored by a rather small size of the system core, with a radius of the outermost closed isobar (ROCI) around $220 \mathrm{~km}$ before $\mathrm{RI}$ in the BT, which is about half the climatological mean ROCI value in the SWIO basin (Leroux et al. 2018). The smallest systems are more prone to undergo rapid intensity changes due to their stronger sensitivity to the synoptic environment (Leroux et al. 2018).

Although the rapid intensification of VITC Hellen was not well anticipated by NWP models, it was supported by a generally conducive environment, especially aloft. The upper-level conditions were indeed particularly favorable for TC intensification, with a prominent poleward outflow channel fostering strong divergence. This was illustrated by the plume of cirrus (Dvorak 1975) fanning in the southeastern quadrant of the storm (Fig. 1a). The ocean heat content was not a limiting factor, which is not surprising since very warm surface waters are common in the Mozambique Channel yearround (Leroux et al. 2018, see their Fig. 2). However, the sudden decay of the storm could not be explained by the

\footnotetext{
${ }^{4}$ Equivalent to $142 \mathrm{kt}$ or $73 \mathrm{~m} \mathrm{~s}^{-1}$ when considering 1-min average winds following U.S. agencies standards.
} 

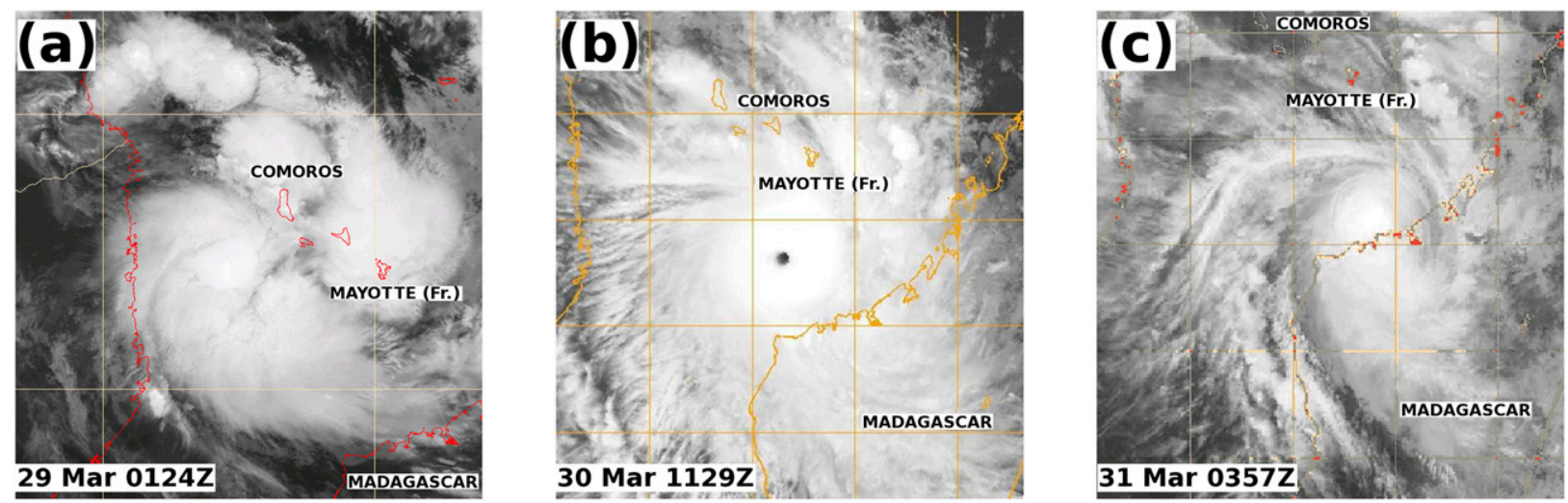

FIG. 1. Naval Research Laboratory (NRL) infrared satellite images at three different stages of Hellen's life cycle. Courtesy of the NRL tropical cyclone satellite web page (http://www.nrlmry.navy.mil/TC.html). (a) Development stage, at 0124 UTC 29 Mar 2014 (satellite platform: NOAA-18), best track maximum winds: $40 \mathrm{kt}\left(20 \mathrm{~m} \mathrm{~s}^{-1}\right)$. (b) Maturity stage, at 1129 UTC 30 Mar 2014 (satellite platform: F15), best track maximum winds: $120 \mathrm{kt}\left(62 \mathrm{~m} \mathrm{~s}^{-1}\right)$. (c) Decay stage, at 0357 UTC 31 Mar 2014 (satellite platform: F18), best track maximum winds: $60 \mathrm{kt}\left(31 \mathrm{~m} \mathrm{~s}^{-1}\right)$.

typical TC intensity predictors that the forecasters follow routinely (e.g., 200-850-hPa VWS, 200-hPa divergence, sea surface temperatures). This contributed to the failure in the intensity prediction of this system.

\section{b. Available observations and reanalysis data}

BT data are mainly based on Dvorak estimates in the SWIO basin. These estimates give less reliable results than direct airborne observations, as available in the northeastern Pacific and North Atlantic basins. Note that, at the end of VITC Hellen's rapid weakening period, the BT takes into account a bull's-eye ASCAT METOP-B (Figa-Saldaña et al. 2002) swath at 0644 UTC 31 March that confirmed that the VMAX did not reach tropical cyclone intensity anymore, approximately $9 \mathrm{~h}$ before landfall. Additionally, Knaff et al. (2010) provide an evaluation of the Dvorak-based intensity estimates using an Atlantic sample. Although transposition to other basins like the SWIO should be done with care due to regional adaptations of the Dvorak technique (Velden et al. 2006), meaningful indications of Dvorak biases can still be extrapolated from this study. When compared to in situ observations, the root-mean-square errors of Dvorak estimates depend primarily on the intensity of the system, with the Dvorak estimates being more precise for the $90-125-\mathrm{kt}$ range $\left(46-64 \mathrm{~m} \mathrm{~s}^{-1}\right)$. When considering the most rapidly intensifying systems through the highest 12-h intensity trends, the Dvorak technique appears to underestimate the intensity for all systems, but especially for category 5 storms. By recommending not to strictly abide by the Dvorak constraints on intensity changes, especially in RI cases, Knaff et al. (2010) also gave credibility to the RSMC forecasters' operational estimations and to the BT data that break those constraints. This conscious infringement aims at reducing the aforementioned negative bias observed in RI cases.

As shown hereafter, the presence of a $400-\mathrm{hPa}$ environmental constraint, associated to unsaturated air, was most likely critical in triggering the storm decay. However, few observations were acquired during the lifetime of VITC Hellen to confirm the presence of the rather thin dry-air layer described in the experimental simulation. Despite a coarse horizontal resolution $(80 \mathrm{~km})$, the ERA-Interim reanalysis dataset (Dee et al. 2011) nevertheless gives a good indication of the broad environmental conditions surrounding Hellen (Fig. 2) at 0000 UTC 31 March. The moist envelope of the system is visible in Fig. 2a. Dry air, associated to relative humidity lower than $40 \%$, is located west of the storm and less than $300 \mathrm{~km}$ away from the storm center. Braun et al. (2012) suggested that the presence of environmental midlevel dry air may reduce the size of the storm; this could explain the rather small size of VITC Hellen. A vertical profile of relative humidity located relatively close to the BT center indicates that the dry layer is rather thin and centered around the $400-\mathrm{hPa}$ level in the ERA-Interim reanalysis (Fig. 2b). The same profile in the AROME-IO simulation, averaged on a $80-\mathrm{km}$ disk to be compared with the ERA-Interim data, compares well with the reanalysis (Fig. 2b). The main difference between AROME-IO and ERA-Interim appears to be the thickness of the dry-air layer, with a shallower structure in AROME-IO.

\section{c. Model configuration}

Since February 2016, a new nonhydrostatic highresolution NWP system has been run in operations at 


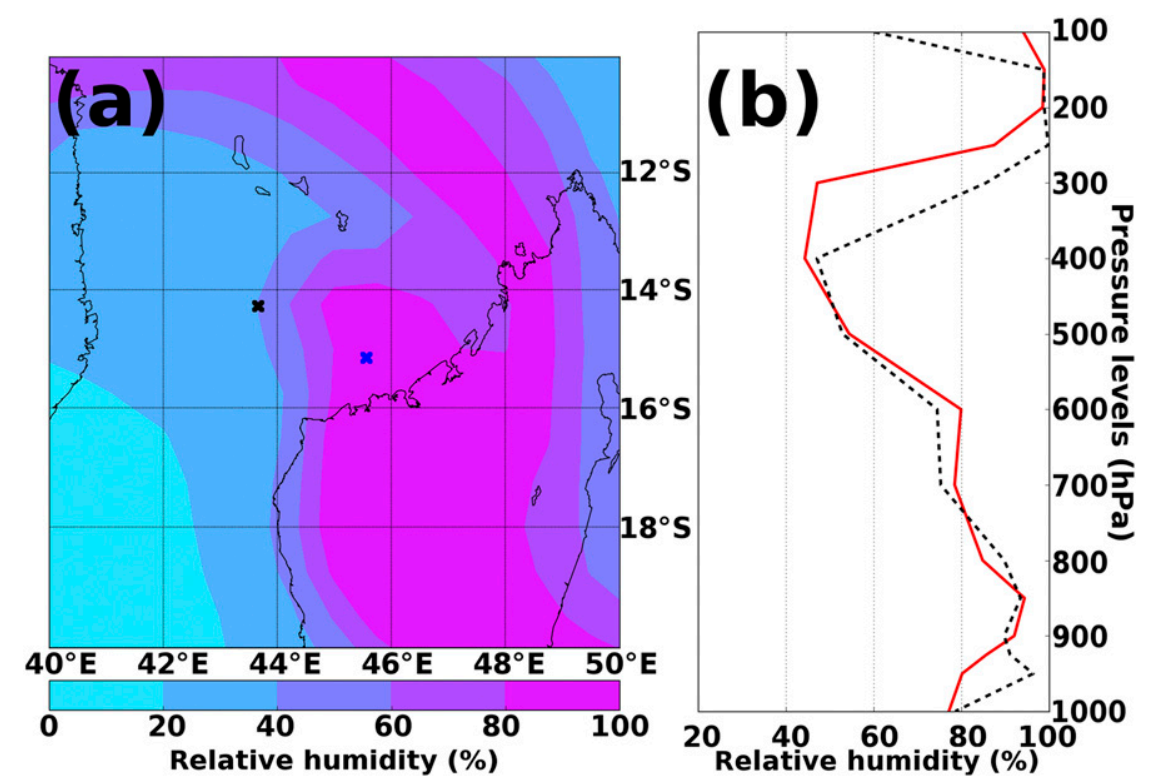

FIG. 2. Spatial and vertical distributions of relative humidities (\%) in the environment of VITC Hellen at lead time $24 \mathrm{~h}$ (0000 UTC $31 \mathrm{Mar}$ ). (a) At $400 \mathrm{hPa}$ from ERA-Interim reanalysis data. The best track TC center is indicated by the blue cross. (b) Vertical profile at the location indicated by the black cross on Fig. 2a from ERA-Interim (red line) and AROME-IO experiment averaged on a $40-\mathrm{km}$ disk in order to compare to the $80-\mathrm{km}$ resolution of the ERAInterim reanalysis data (dashed black line).

RSMC La Réunion to improve both TC prediction and weather forecasts at the convective scale (Faure et al. 2018). This model, named AROME-IO, is largely based on the French model AROME (Seity et al. 2011), but is adapted to the context of the SWIO region (Table 1). With a $2.5-\mathrm{km}$ horizontal grid and 90 vertical levels (beginning at $5 \mathrm{~m}$ ), AROME-IO allows an explicit representation of deep convection. It includes an updated version of AROME's three-class ice parameterization microphysical scheme ICE3 (Pinty and Jabouille 1998). Part of the setup consists of a dynamical adaptation of the Integrated Forecasting System (IFS) from the European Centre for Medium-Range Weather Forecasts (ECMWF). The initial and lateral boundary conditions are provided by downscaled ECMWF IFS atmospheric fields. At the initial time, the ocean state is given by an analysis from the global model PSY4 (Mercator-Ocean) analysis based on the NEMO ocean code (Madec 2008) and held constant afterward. Thus, the current model setup does not take into account the retroactive processes between the TC and the ocean, such as the upwelling caused by the cyclonic circulation at the sea surface. Nevertheless, the model is able to reproduce the rapid weakening of the storm (Fig. 3a), suggesting that the ocean feedback is not likely playing a dominant role in the rapid weakening of VITC Hellen. Furthermore, the ocean analysis used in this simulation indicates a very high ocean heat content over the whole area (not shown) ranging from 75 to $125 \mathrm{~kJ} \mathrm{~cm}^{-2}$, which is enough to fuel a VITC moving at a mean $5-\mathrm{kt}$ $\left(2.5 \mathrm{~m} \mathrm{~s}^{-1}\right)$ speed.

To investigate the rapid weakening of VITC Hellen, an experiment based on AROME-IO has been run over a restricted geographical area covering the northern half of the Mozambique Channel (from $10.75^{\circ}$ to $25.5^{\circ} \mathrm{S}$ and from $42.5^{\circ}$ to $60^{\circ} \mathrm{E}$ ). The simulation begins at 0000 UTC 30 March 2014 and ends $72 \mathrm{~h}$ later, at 0000 UTC 2 April 2014. This study focuses on the first $48 \mathrm{~h}$ of the simulation. Results during the first $6 \mathrm{~h}$ will be discarded owing to the model spinup.

\section{d. Model validation}

The simulated track, intensity and structure are verified against available observations and BT data. Figure 3a compares the minimum sea level pressure

TABLE 1. AROME-IO model characteristics.

\begin{tabular}{ll}
\hline \hline Dynamics & $2.5 \mathrm{~km}$ L90 (5 m), spectral, nonhydrostatic \\
Microphysics & ICE3 (Pinty and Jabouille 1998) \\
Deep convection & Explicit \\
Shallow convection & PMMC09 (Pergaud et al. 2009) \\
Boundary conditions & ECMWF HRES (1 way) \\
Forecast range & $72 \mathrm{~h}$ \\
\hline
\end{tabular}



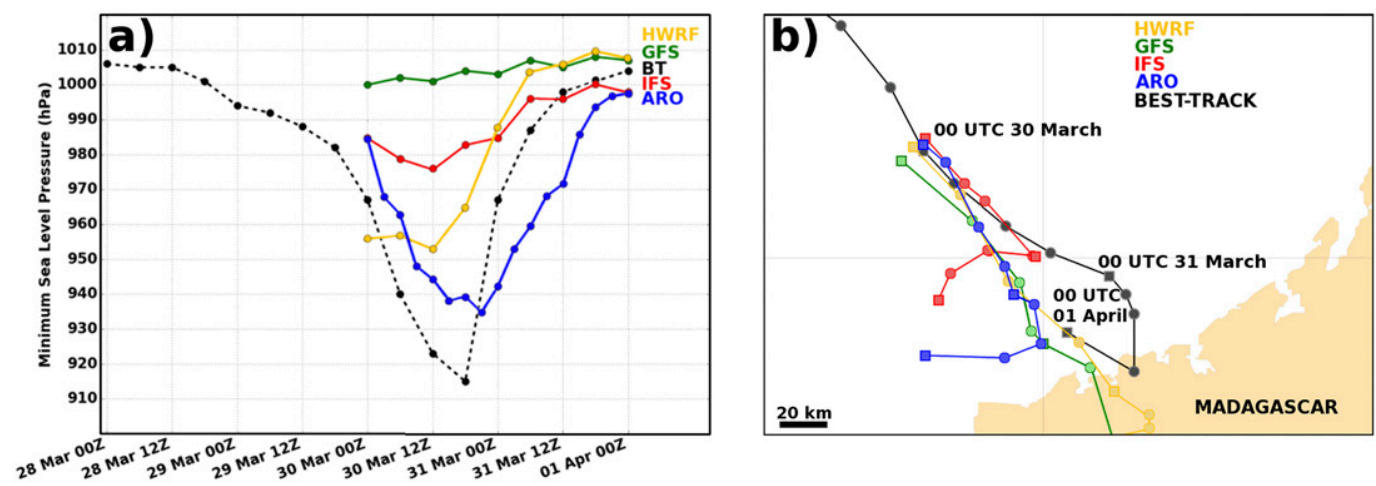

FIG. 3. Comparison between the available operational guidance over $72 \mathrm{~h}$ from base time 0000 UTC $30 \mathrm{Mar}-$ GFS (green line), IFS (red line), HWRF (gold line)—and the AROME-IO experiment (blue line) for VITC Hellen. The best track data are plotted for reference (black line). (a) Central pressure (hPa) and (b) track (squares are for 0000 UTC times and circles delineate other synoptic times).

(MSLP) forecasts of AROME-IO and the operational GFS, IFS, and $\mathrm{HWRF}^{5}$ models from base time 0000 UTC 30 March 2014 with the BT data. At 0000 UTC 30 March, the MSLP in the GFS model analysis is some $30 \mathrm{hPa}$ higher than the BT estimate. With such a biased initial state, the operational GFS run is not able to correctly reproduce Hellen's intensification. The IFS analysis, that initializes AROME-IO, is closer to the BT but remains some $18 \mathrm{hPa}$ higher. The IFS forecast has a good timing for the deepening, maximum intensity and weakening of the storm, but with a 6-h lag and much more reduced intensity changes. The HWRF analysis is closer to the BT than any of the other three models. This is mainly because the initial state of HWRF uses the Joint Typhoon Warning Center (JTWC) storm messages to adjust the storm position, structure and intensity. The HWRF model only slightly deepens the low until 1200 UTC 30 March before beginning its weakening phase $6 \mathrm{~h}$ too early compared to the BT. The HWRF simulated storm makes landfall between 1800 UTC 30 March and 0000 UTC 31 March (Fig. 3b), which means that the rapid weakening occurring after 1800 UTC 30 March is strongly driven by direct land interaction and not representative of Hellen's oversea weakening.

In the first 6-h spinup, the AROME-IO vortex quickly reaches the VITC intensity, with maximum winds exceeding $115 \mathrm{kt}\left(59 \mathrm{~m} \mathrm{~s}^{-1}\right)$. The peak intensity of the storm by the model is predicted at 2100 UTC 30 March by the model, with a 3-h delay compared to the BT data (6-h resolution). Even if the predicted minimum sea level pressure has a positive 24-hPa bias compared to the

\footnotetext{
${ }^{5}$ Hurricane Weather Research and Forecasting system, developed and run by the National Oceanic and Atmospheric Administration (NOAA).
}

BT data, the AROME-IO prediction is more skillful than that of the operational IFS (+51-hPa bias), GFS $(+85 \mathrm{hPa})$, and HWRF $(+38 \mathrm{hPa})$ models. More significant is the fact that the experimental model reproduces the fast weakening while never making landfall, albeit with a 3-h lag compared to the BT (Fig. 3b). The AROME-IO simulated TC center is closest to the coast at 1200 UTC 31 March $(32 \mathrm{~km})$.

In the following, the simulated TC center will be tracked using the minimum sea level pressure in the model. The track forecast of AROME-IO is relatively skillful with direct position errors (DPE) remaining under $100 \mathrm{~km}$ during the first $36 \mathrm{~h}$ (maximum error of $89 \mathrm{~km}$ ). As a comparison, at forecast lead time (hereafter abbreviated as lead time) $36 \mathrm{~h}$, the IFS predicted center is located some $146 \mathrm{~km}$ away from the BT center while the GFS and HWRF centers are 117 and $110 \mathrm{~km}$ away, respectively, and already over land. The IFS and GFS errors are lower than the mean DPE value of these models for the basin around that lead time and over the 2004-14 period (Leroux et al. 2016, see their Table 1).

The simulated vortex is compared to the structure observed on microwave images retrieved by radiometers of low-orbiting satellites. First, the focus is on the simulated structure of VITC Hellen at peak intensity with an intercomparison of real and synthetic radar rain rate (Fig. 4). Rainfall measurements are only available for the northeastern half of the TC (Fig. 4a), so the $37-\mathrm{GHz}$ microwave radiometer data was chosen to cover the remaining semicircle. The positioning error is low as the predicted TC center in the simulation remains close to the position observed on the microwave image (Fig. 4a). In the simulation, the strongest rain rates are located in the southwestern quadrant of the eyewall (Fig. 4b, black polygon). Interestingly, this corresponds to the most intense convective spots in TRMM data. Spiraling convective 

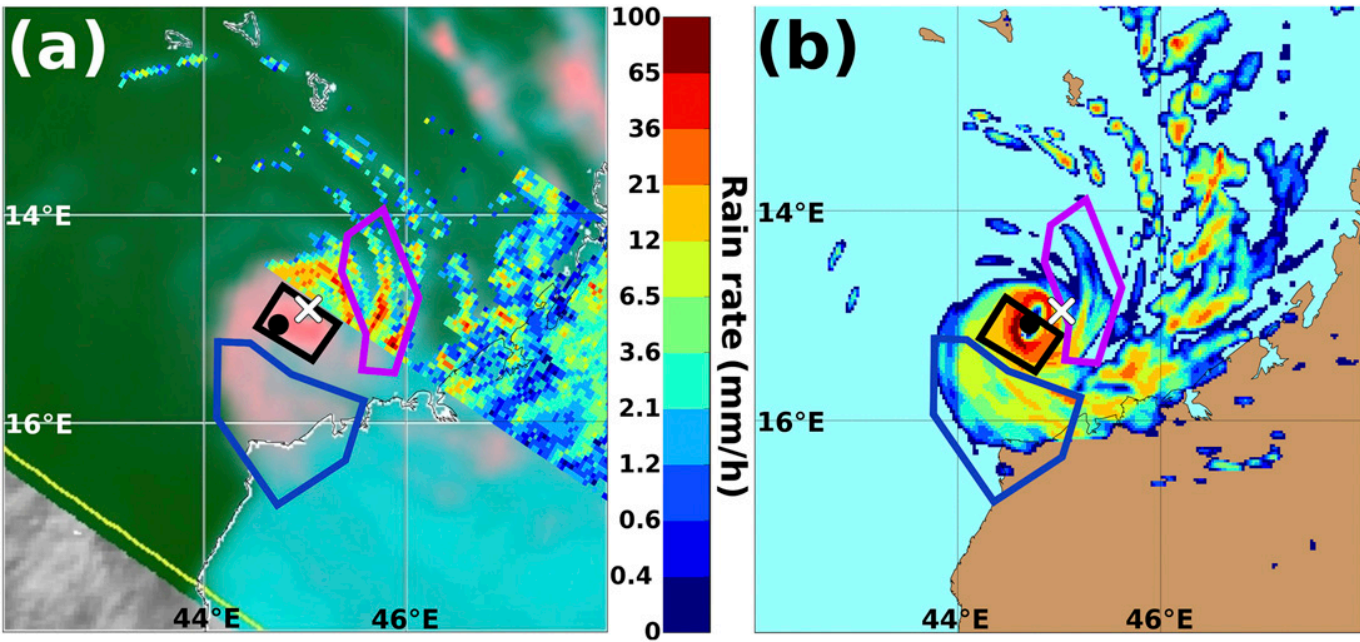

FIG. 4. Validation of the AROME-IO experiment by intercomparison with satellite data during VITC Hellen's peak intensity. The bold polygons delimit the areas being investigated. The black dot shows the position of the TC center in the simulation, while the white cross shows the analyzed position derived from satellite data. Map grid spacing is $2^{\circ}$. (a) Rain estimates $\left(\mathrm{mm} \mathrm{h}^{-1}\right.$ ) from the TRMM-2B31 algorithm (Kummerow et al. 1998) overlaid on NRL 37 color image (37-GHz color composite) from the TRMM overpass at 1745 UTC 30 Mar 2014. Courtesy of the NRL TC satellite web page (http://www.nrlmry.navy.mil/TC.html). (b) 500-m rain rate $\left(\mathrm{mm} \mathrm{h}^{-1}\right)$ at 1800 UTC 30 Mar from AROME-IO forecast. The rain data share the same color scale on both (a) and (b).

bands are also well reproduced by the model, such as one of the most active bands close to the center (magenta polygon). Finally, the asymmetry in the storm large-scale structure is correctly reproduced, with a larger extension of convection in the southern semicircle (blue polygon). The main features of VITC Hellen's core around peak intensity are therefore quite accurately reproduced by the AROME-IO experiment.

To validate the skill of the model during the rapid decay period of VITC Hellen, the SSMIS 89-GHz image is compared to simulated graupel fields at $500 \mathrm{hPa}$ (Fig. 5). The use of a simulated microwave image would have been more appropriate for such a comparison, but the current radiative scheme of AROME-IO is not yet able to output this type of data. Given that the microwave images are used in the operational context as a proxy for the density of hydrometeors, the comparison proposed in Fig. 5 is still relevant. At this time, the positioning error increases a little, as the predicted circulation center is too far west and thus a bit too far from the Madagascan coastline. As seen on Fig. 5a, the most intense convective processes are still located in the southern half of the eyewall (blue polygon). A similar asymmetry is found in the simulated eyewall thunderstorm activity through higher graupel concentrations (Fig. 5b). The southern outer semicircle (magenta polygon) is also well reproduced, with less activity than in the eyewall, but much deeper convection than in the northern half of the TC. In this area, the convective activity is most likely supported by the solid poleward outflow channel causing very strong upper divergence. Last, the focus is on peripheral rainbands (black polygon). The model simulates too much thunderstorm activity south of the Comoros archipelago, but is still relatively close to the observed situation. Considering that this comparison is taking place at lead time $27 \mathrm{~h}$, the AROME-IO forecast drift remains limited compared to the observed center, showcasing the model's ability to provide meaningful positioning guidance.

As shown above, the evolution of the VITC core is well reproduced during peak intensity and during its rapid weakening over sea.

\section{Results}

\section{a. Environmental conditions at peak intensity}

$\mathrm{TC}$ forecasters are used to evaluating the influence of the environmental VWS by monitoring the wind difference between 200 and $850 \mathrm{hPa}$ (Fig. 6a), corresponding to the deep-layer VWS. In the case of this simulated storm, the $850-200-\mathrm{hPa}$ VWS at peak intensity (lead time $21 \mathrm{~h}$ ) is imposing only a light constraint on the TC structure, with the strongest VWS located in the southwestern quadrant. The wind is not uniform on the area with almost no VWS in the northeastern semicircle. With a $9 \mathrm{~m} \mathrm{~s}^{-1}$ northwesterly wind averaged over the 150-500-km annulus (Fig. 6a), the upper-level environmental conditions appears to be slightly unfavorable for a VITC. A strong poleward 

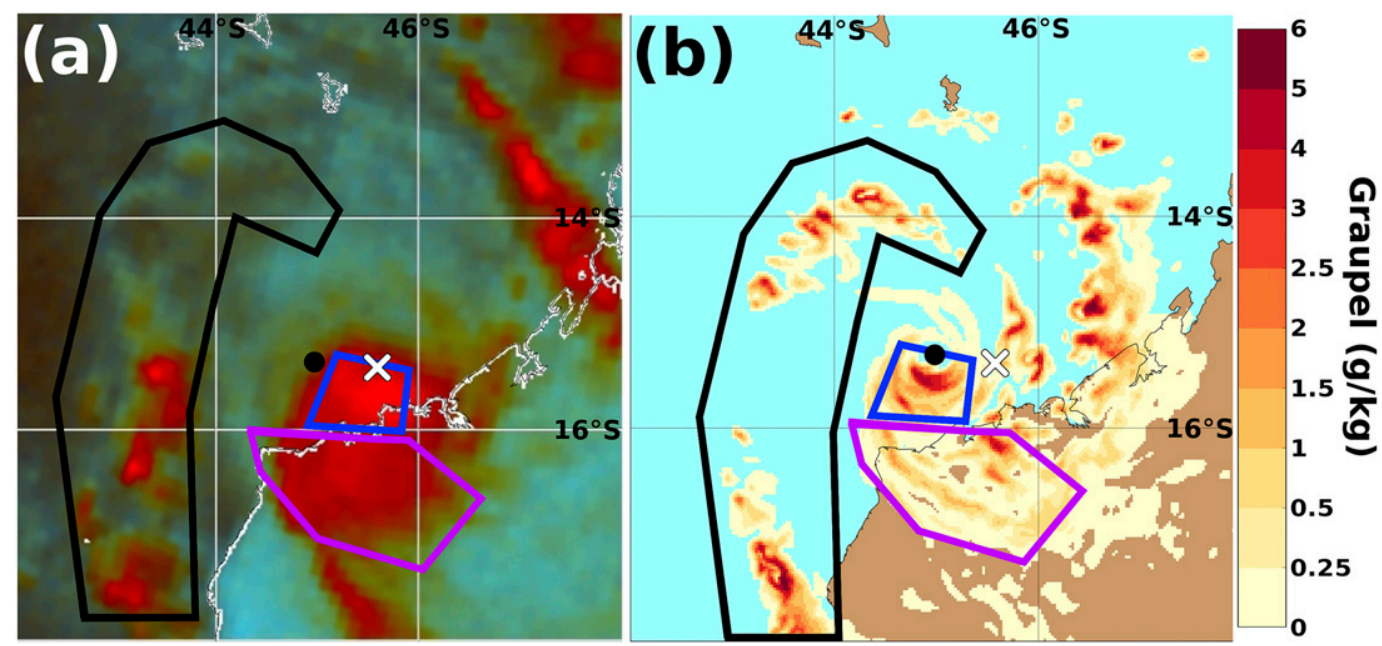

FIG. 5. Validation of the AROME-IO experiment by intercomparison with satellite data during VITC Hellen's rapid weakening. The bold polygons delimit the areas being investigated. The black dot shows the position of the TC center in the simulation, while the white cross shows the analyzed position derived from satellite data. Map grid spacing is $2^{\circ}$. (a) NRL 89 color image (89-GHz color composite) from an F17 SSMIS overpass at 0245 UTC 31 Mar 2014. Courtesy of the NRL TC satellite web page (http://www.nrlmry.navy.mil/TC.html). (b) 500-hPa graupel $\left(\mathrm{g} \mathrm{kg}^{-1}\right)$ at 0300 UTC 31 Mar from AROME-IO forecast.

outflow channel is also located in the southeastern quadrant. The associated 200-hPa relative humidity field does not show any significant pattern or humidity depletion that could affect a mature TC, with mainly saturated air over the area. A drier region is located far from the center in the southwestern quadrant and the 200-hPa environmental wind appears to be moderate in Fig. $6 \mathrm{c}\left(5 \mathrm{~m} \mathrm{~s}^{-1}\right)$. The environmental wind is an average over a $150-500-\mathrm{km}$ annulus with the simulated storm motion removed from the calculation (the choice of radii is discussed in section 4 ).

At $400 \mathrm{hPa}$, the environment is less favorable for TC development (Fig. 6b). The 850-400-hPa VWS is imposing a northwesterly constraint on the storm, with values exceeding $40 \mathrm{kt}\left(21 \mathrm{~m} \mathrm{~s}^{-1}\right)$ in the vicinity of the core and a mean value over the annulus of $10 \mathrm{~m} \mathrm{~s}^{-1}$. Associated with the strong 850-400-hPa VWS, remarkably low humidity is also surrounding the TC core, especially in the western semicircle. The lowest humidity values are less than $20 \%$, and closest to the center where the $400-850-\mathrm{hPa}$ VWS is the strongest. To put these values into perspective, an area-averaged ERAInterim climatology computed over the last 20 years for the month of March over the northern half of the Mozambican Channel $^{6}$ provides a mean relative humidity of $48 \%$ at $400 \mathrm{hPa}$. The climatological minimum of relative humidity is also located at the $400 \mathrm{hPa}$. The midtropospheric VWS constraint may explain the strong asymmetry

\footnotetext{
${ }^{6} \mathrm{On}$ a box from $11^{\circ}$ to $15^{\circ} \mathrm{S}$ and from $41^{\circ}$ to $46^{\circ} \mathrm{E}$.
}

patterns previously presented in Figs. 4 and 5, that resemble to the stationary band complex described by Willoughby et al. (1984). The strong upper-level divergence located in the southern semicircle, and likely caused by the outflow channel may also play a role in this asymmetry. However, the midlevel dry-air layer is rather thin in the simulation, as no similar pattern is found at $600 \mathrm{hPa}$ or below (Fig. 2b). Even though this structure may be able to slow down or impede the convection in the northwestern quadrant of the storm through midlevel ventilation (Tang and Emanuel 2010), it is most likely not the sole mechanism responsible for the abrupt weakening of the storm.

Finocchio et al. (2016) explored the effects of a wide range of VWS vertical structures on idealized TCs and obtained very diverse responses. These results suggest that the commonly used 200-850-hPa VWS metric may not be sufficient to completely understand the effects of VWS on TCs. Shallower and lower VWS, especially, may be more destructive for TCs. Here, the vertical profile of environmental wind (Fig. 6c) shows a discrepancy between the 300-500-hPa layer and the upper levels located above $300 \mathrm{hPa}$. The height of the maximum VWS is located at $400 \mathrm{hPa}$ and extends within a rather shallow (200-hPa width) layer. This structure is not entirely captured by the deep layer shear metric, and this could partly explain why the impact of the VWS was not well assessed by operations. Additionally, Finocchio and Majumdar (2017) provide climatologies of VWS structure over the Northern Hemisphere tropics. Although some differences are shown between the considered basins, the 

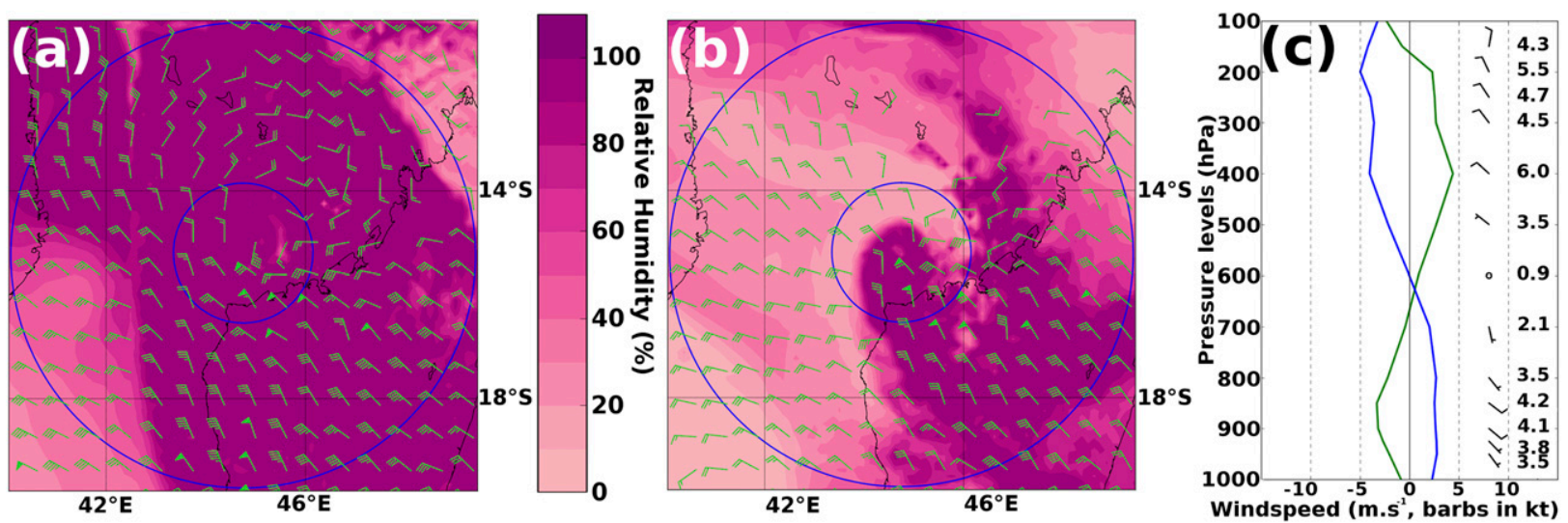

FIG. 6. Environment of the simulated TC at peak intensity [i.e., at lead time $21 \mathrm{~h}$ (2100 UTC 30 Mar)]. Relative humidity (\%, shaded) and wind shear with respect to $850 \mathrm{hPa}$ greater than $10 \mathrm{kt}$ (green barbs in kt) at (a) 200 and (b) $400 \mathrm{hPa}$. (c) Vertical profile of the stormrelative zonal (green line, $\mathrm{m} \mathrm{s}^{-1}$ ), meridional (blue line, $\mathrm{m} \mathrm{s}^{-1}$ ), and total (black barbs, kt, the strength in $\mathrm{m} \mathrm{s}^{-1}$ is given by the black number in the left column) environmental winds averaged on a 150-500-km annulus delineated by the blue circles on (a) and (b).

mean zonal winds exhibit similar vertical structures, with wind speeds increasing with altitude. With its 400-hPa maximum, the environmental wind of VITC Hellen exhibits an unusual vertical structure. The storm-relative environmental wind also becomes zero at $600 \mathrm{hPa}$, indicating the level of the steering flow for the simulated storm.

Riemer and Montgomery (2011) explored the combinative effects of environmental wind and dry air on a TC through a simple kinematic model. Although dry environmental air is advected closer to the TC core by the environmental flow, the swirling winds of the TC vortex strongly deflect the cross-vortex flow. The TC is thus able to isolate itself from adverse thermodynamic interaction. However, Riemer and Montgomery (2011) have identified a well-defined source region of environmental dry air, depending on the direction of the environmental wind. In Hellen's case, with a Southern Hemisphere TC vortex (thus a clockwise rotation) embedded in a northwesterly environmental wind at $400 \mathrm{hPa}$, the source region should be located preferentially in the southwestern quadrant. In this region, the air is particularly dry compared to the eastern semicircle (Figs. 2a, 6b). Riemer and Montgomery (2011) provide a regime diagram for the environmental interaction of TCs in VWS, depending on the strengths of the TC circulation and of the background flow. Using this diagram with a category 5 vortex and considering a $6 \mathrm{~m} \mathrm{~s}^{-1} 400-\mathrm{hPa}$ storm-relative environmental flow (Fig. 6c), results from Riemer and Montgomery (2011) suggest a regime of "band interaction" for Hellen and indicate that the environmental wind is not strong enough to cause a direct eyewall interaction. However, this diagram was derived from experiments where the ideal TC vortex was based on average TC features and based upon the location of their "dividing streamline" that is largely dependent on the size of the cyclonic circulation and not only the strength of the environmental constraint. With a BT ROCI two times smaller than average over the basin and a simulated RMW lower than $20 \mathrm{~km}$ on a large part of the period of interest, Hellen is thus expected to be less able to thermodynamically isolate itself from the environment (Riemer and Montgomery 2011). Consequently, the regime diagram is expected to overestimate the resiliency of VITC Hellen and the eyewall region may still be affected by environmental air. These results suggest that the synoptic configuration at $400 \mathrm{hPa}$ allows a direct interaction between the environmental midlevel dry air and the TC.

\section{b. Low-level processes}

The thermodynamic mechanisms describing the interactions between TCs and environmental VWS (Onderlinde and Nolan 2017; Riemer et al. 2010) are examined hereafter by computing the downward advection of cooler $\theta_{e}$ air (cooler $\theta_{e}$ downward advection, hereafter referred to as CTDA) in the inflow layer (defined hereafter by the layer comprised between the surface and $850 \mathrm{hPa}$ ):

$$
\mathrm{CTDA}=-w \frac{\partial \theta_{e}}{\partial z} \quad \text { with } \quad w<0 \text { and } \quad \frac{\partial \theta_{e}}{\partial z}<0,
$$

where $w$ is the vertical wind speed.

It should be noted that, by definition, CTDAs are always negative as they result in a decrease of $\theta_{e}$ values within the inflow layer. It should be noted that this formulation differs from the original diagnosis of Riemer et al. (2010) in that a downward advection is preferred here to the downward flux of low- $\theta_{e}$ (DFX). The latter diagnosis considers a deviation $\left(\theta_{e}^{\prime}\right)$ air from the azimuthal 

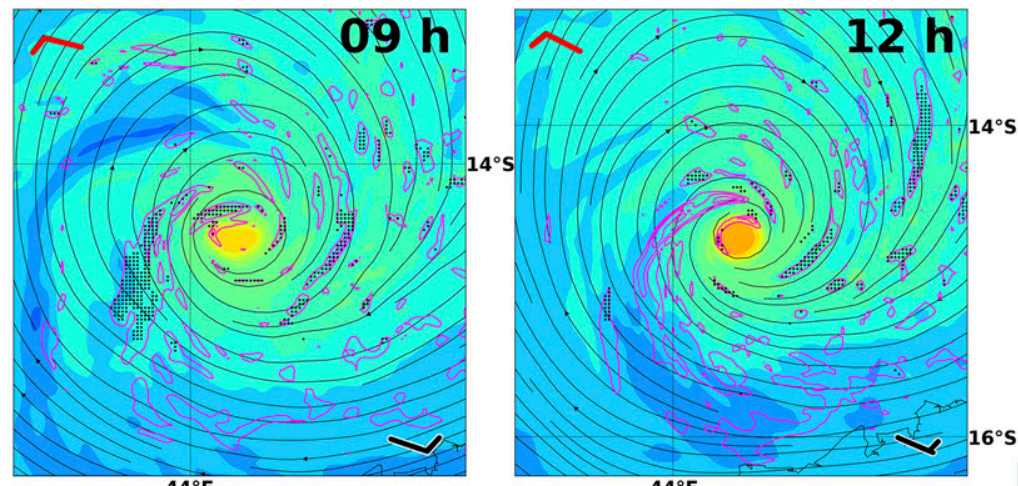

$14^{\circ} \mathrm{S}$
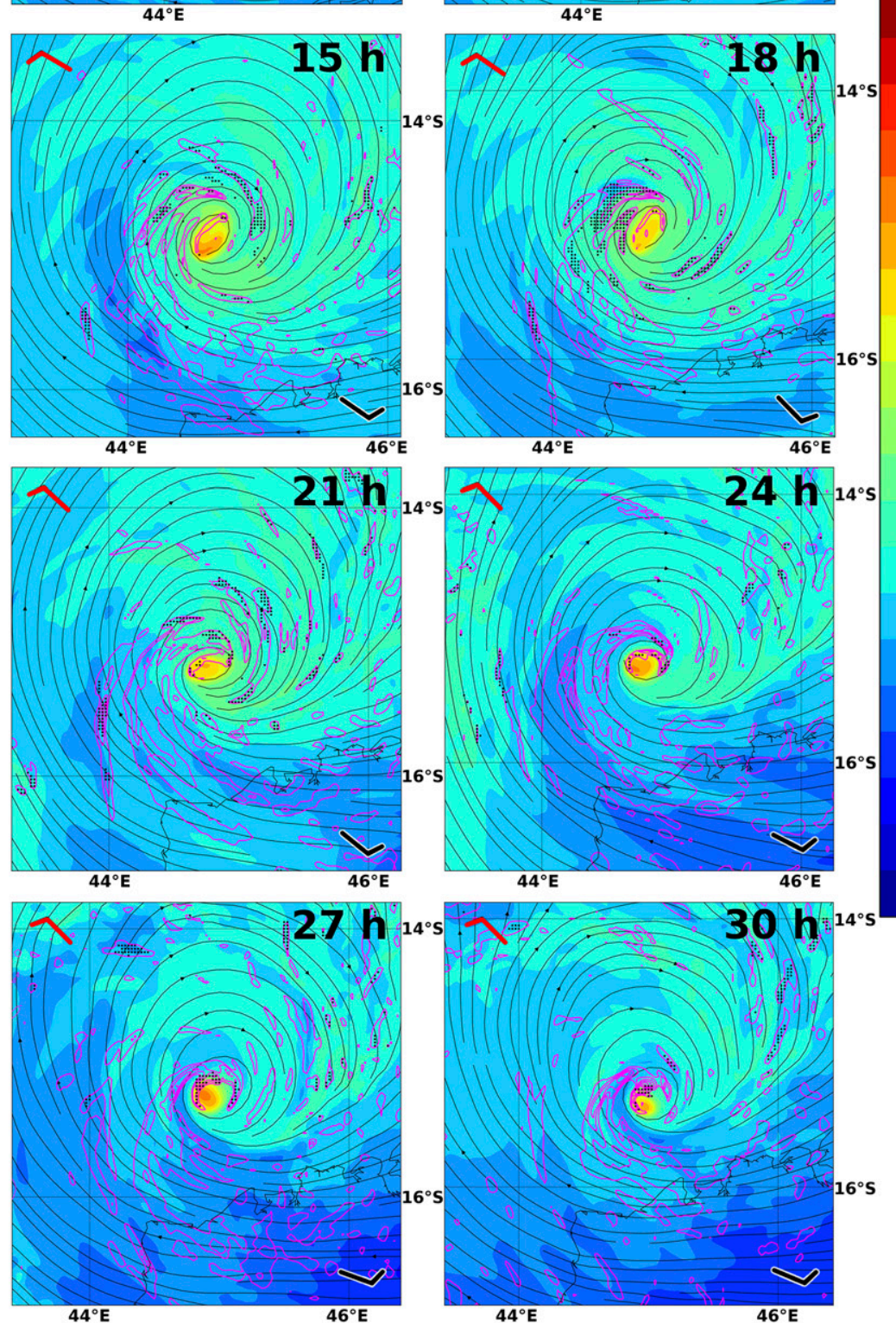

$44^{\circ} \mathrm{E}$
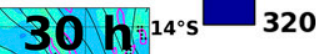

330

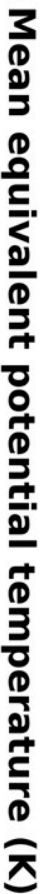

FIG. 7. Evolution of Hellen's inflow layer from lead time $9 \mathrm{~h}$ ( 0900 UTC 30 Mar) to lead time $30 \mathrm{~h}$ (0600 UTC 31 Mar) with a 3-h time step, in the AROME-IO experiment. Indicated are mean $\theta_{e}$ values between 925 and $850 \mathrm{hPa}$ (K, shaded) and downward vertical velocities (purple isolines, from $0.4 \mathrm{~m} \mathrm{~s}^{-1}$ every $\left.1 \mathrm{~m} \mathrm{~s}^{-1}\right)$. Black dotted areas delimit regions where the mean cooler $\theta_{e}$ downward advections at 
mean to investigate the asymmetries induced by the VWS. However, in a nonidealized case where the environment is not controlled, $\theta_{e}$ asymmetries may not be only related to the action of VWS on the TC. In Hellen's case, the presence of the Madagascan landmass relatively close-by is likely to induce additional asymmetries, especially through horizontal advection. To isolate the contribution of downward intrusions of low- $\theta_{e}$ air, we chose to look at a vertical difference of $\theta_{e}$ rather than an axisymmetric deviation of $\theta_{e}$ on a single level. Additionally, Riemer et al. (2010) noted that an axisymmetric viewpoint may not be ideal in the case of strong asymmetries within the TC, which is the case here (oval shape of the highest $-\theta_{e}$ area representative of the TC inner core in Fig. 7, at 15 and $18 \mathrm{~h}$ ).

The evolution of the mean $\theta_{e}$ in the inflow layer is presented in Fig. 7, along with downward velocities and CTDAs at the top of the inflow layer $(850 \mathrm{hPa})$. The TC core is well delimited by the highest- $\theta_{e}$ (yellow shades) while lower- $\theta_{e}$ intrusions are delimited by blue shades. Downward motion at the top of the inflow layer is occurring preferentially in the western semicircle and closer to the TC center in the northwestern quadrant (i.e., upshear) at all lead times. This quasi-stationary structure is presenting a clear azimuthal wavenumber-1 asymmetry that is reminiscent of the DFX pattern presented in Fig. 5 of Riemer et al. (2013). Between 15 and $24 \mathrm{~h}$, the downward velocity cores are gradually coming closer to the simulated TC center. The strongest patterns are seen in the northwestern quadrant at $18 \mathrm{~h}$, with almost all of the downward velocity cores participating in CTDAs, collocated to lower $\theta_{e}$ than the close environment of the TC. From lead time $21 \mathrm{~h}$ onward, downward velocities associated to CTDAs are located relatively close to the core and as the rapid weakening begins, these downdrafts maintain very close to the TC inner core. CTDAs are occurring inside the radius defined by the highest $-\theta_{e}$ values.

The perimeter of the central area delimited by the highest $\theta_{e}$ can be considered as the so-called limit cycle described in Riemer and Montgomery (2011), that delimits an area in their kinematic model in which layer-wise advection into the TC inner core from the environment is impossible because of the strong deflection by the TC vortex. This central area has an $18-20-\mathrm{km}$ radius, while the simulated RMW remains close to $16 \mathrm{~km}$ throughout the period of interest. As seen above, CTDAs are occurring at every time step from lead time $21 \mathrm{~h}$ inside this inner ring that is theoretically unreachable for layer-wise advection. In the inflow layer, the mean storm-relative environmental wind is southeasterly (Fig. 7) and thus, the model of Riemer and Montgomery (2011) indicates the northeastern quadrant as the preferential source region of environmental air for a Southern Hemisphere TC. Within the inflow layer, the lowest $\theta_{e}$ values in the environment of the simulated storm are located over the Madagascan landmass in the southeastern quadrant while relatively high $\theta_{e}$ values are displayed in the northeastern region (Fig. 7). The relative humidities of the ERA-Interim climatology within the 1000-850-hPa layer are $5 \%$ to $10 \%$ lower on the Madagascan landmass compared to the mean values computed over the whole region. Following Riemer and Montgomery (2011), the low $-\theta_{e}$ air of the Madagascan landmass should not directly interact with the TC core as long as the TC remains relatively far enough from the coast. Between 12 and $18 \mathrm{~h}$, low $-\theta_{e}$ values are only wrapping around the southwestern quadrant while remaining much farther from the center than the CTDAs (Fig. 7). The environmental wind streamlines show that the air coming from Madagascar is deflected to the western semicircle relatively far from the center. However at $21 \mathrm{~h}$, just after the 18-h maximum CTDAs and as the TC gets closer to the coast, lower- $\theta_{e}$ air begins to wrap tighter around the TC core than on the previous lead times. However, the streamlines originating from the Madagascan landmass do not directly reach the TC inner core. At $24 \mathrm{~h}$, the weakening phase of the simulated TC has already begun and relatively low $\theta_{e}$ air surrounds the TC core in all but the southeastern quadrant. At this time, the topology of the flow shows that the streamlines originating in the northeastern quadrant lead directly to the inner core while the ones from the southeastern quadrant form a complete spiral before reaching the inner region. The downward velocity signal is also strong at $21 \mathrm{~h}$ but as the $\theta_{e}$ air in the inflow layer has already been depressed, CTDAs are not as visible because of a lower- $\theta_{e}$ difference between the inflow layer and the layers above. From lead time $27 \mathrm{~h}$, the cooler Madagascan air participates more directly to the depression of the $\theta_{e}$ close to

\footnotetext{
$850 \mathrm{hPa}$ are lower than $-5 \mathrm{~K} \mathrm{~h}^{-1}$. Black streamlines indicate the mean environmental flow at $900 \mathrm{hPa}$ after removing the simulated storm motion. The storm-relative winds computed at 400 and $900 \mathrm{hPa}$ and averaged on a $150-500-\mathrm{km}$ annulus are indicated by wind barbs $\left(\mathrm{kt}, 1 \mathrm{kt}=0.5144 \mathrm{~m} \mathrm{~s}^{-1}\right)$ located in the upper left (red barb, $400 \mathrm{hPa}$ ) and lower right (black barb, $900 \mathrm{hPa}$ ) corners. Plots are centered on the simulated vortex center.
} 
the TC center. This is coherent with Riemer and Montgomery (2011) theory because once the simulated TC has come close enough to the coast, the lower- $\theta_{e}$ air of the southeastern quadrant may now reside within the so-called dividing streamline that encompasses the area in which the environmental air is attracted close to the TC core by the low-level inflow in all sectors. Because of its small size, TC Hellen may have a closer "dividing streamline" than TCs with broader circulations, meaning that it could come closer to the coast before being impacted by direct landmass advection.

Another aspect of the interaction between the surface wind fields of the simulated storm and the nearby Madagascan land surface is a progressive enhancement of the inflow within the right and front-right quadrants of the storm, when adapting the results of Wong and Chan (2007) to the Southern Hemisphere. In the case of Hellen, with a southeasterly motion, the surface inflow is expected to gradually increase within the southwestern semicircle as the system comes closer to land. This is caused by the roughness contrast between the sea and land surfaces that induces asymmetries in the wind radial distribution. When the simulated TC begins to weaken, its center is still located approximately $50 \mathrm{~km}$ away from the coast and the TC has a very compact structure (storm-force winds do not reach the coast) limiting the interaction between the clockwise circulation and the land surface. But as the storm comes closer to the coast, these effects are expected to contribute significantly to a stronger radial advection of Madagascan low-level dry air toward the TC inner core, as seen on the last two panels of Fig. 7.

The 925-hPa $\theta_{e}$ anomaly computed with respect to lead time $9 \mathrm{~h}$, when the simulated TC core is well constituted (Fig. 7), is presented for lead times 15 to $24 \mathrm{~h}$ in Fig. 8. At lead times 15 and $18 \mathrm{~h}$, a dipole of positive and negative anomalies is visible on each side of the TC center. Investigating the origin of this dipole through Fig. 7, the comparison of the 9-h and the 15-h panels reveals that this pattern corresponds to the distortion of the core of high $\theta_{e}$ and is related to the oval shape of the TC inner core discussed earlier. This is not clear if this distortion is caused by the environmental VWS constraint or only by inner-core processes. As no CTDAs occur this close to the TC inner core at lead time $15 \mathrm{~h}$ (Fig. 7), these $\theta_{e}$ anomalies are not considered to be a consequence of downward advections. Consequently, the focus is rather on the 4-K negative anomaly located north of the TC center along the $50-\mathrm{km}$ radius at $15-\mathrm{h}$ lead time (Fig. 8). At lead time $18 \mathrm{~h}$, this anomaly has progressed toward the TC center and has strengthened below $-8 \mathrm{~K}$. This directly illustrates the impact of the strongest CTDA cores located within the northwestern quadrant at same lead times (Fig. 7).

At lead time $21 \mathrm{~h}$, the CTDAs occurring directly into the "limit cycle" area delineated on Fig. 7 are linked to a strong negative anomaly displayed very close to the TC center in the northwestern quadrant (Fig. 8). It should be noted that contrary to $6 \mathrm{~h}$ earlier, the shape and orientation of the core of highest $\theta_{e}$ values is very similar between lead times 21 and $9 \mathrm{~h}$ (Fig. 7). Consequently, this $12-\mathrm{K}$ negative anomaly is believed to be mainly induced by CTDAs.

Some negative anomalies linked to the layer-wise advection from the Madagascan landmass are also displayed on Fig. 8. They are visible at lead time $15 \mathrm{~h}$, more than $75-\mathrm{km}$ away from the center in the western semicircle. The influence of the Madagascan advections is increasing from lead time $21 \mathrm{~h}$ as illustrated by the negative $\theta_{e}$ anomaly originating from Madagascar and spiraling in the northern semicircle. At this lead time, both CTDAs and Madagascan horizontal advections are participating in the negative anomalies displayed over the southern semicircle and northwestern quadrant and their effects are difficult to differentiate. As discussed above, the Madagascan layer-wise advection is not yet expected to reach the vicinity of the inner core at this time, thus its influence is most likely very limited inside the $50-\mathrm{km}$ radius. The spiraling pattern is strengthened at lead time $24 \mathrm{~h}$, while negative anomalies delimited by the 8-K shaded contour and originating directly from the Madagascan landmass are coming closer to the TC center, as the low-level flow topology allows for a more direct layer-wise advection of environmental air.

\section{c. Evolution of Hellen's vertical structure}

Figure 9 presents the evolution of vertical structure of the simulated TC in the northwestern quadrant (upshear). The $\theta_{e}$ vertical distribution within the TC presents a climatological minimum located in the midlevels (blue shading in Fig. 9 at 9 h). Consequently, any downward motion above $700 / 600 \mathrm{hPa}$ advects higher $\theta_{e}$ downward. This explains why CTDAs are located exclusively within low levels.

The RMW computed at the $925-\mathrm{hPa}$ level, is used to indicate the approximate location of the base of the eyewall, which corresponds well to the base of the main core of updrafts on every panels of Fig. 9. A strong core of downward velocities is located in the vicinity of the TC center, inside the RMW. This feature corresponds to the downward motion inside the TC eye and is visible throughout all lead times of Fig. 9. Outside of the RMW, some weak downdrafts with associated CTDAs are occurring within the inflow layer at 9 and $12 \mathrm{~h}$. These downdrafts located between the RMW and the $75-\mathrm{km}$ 

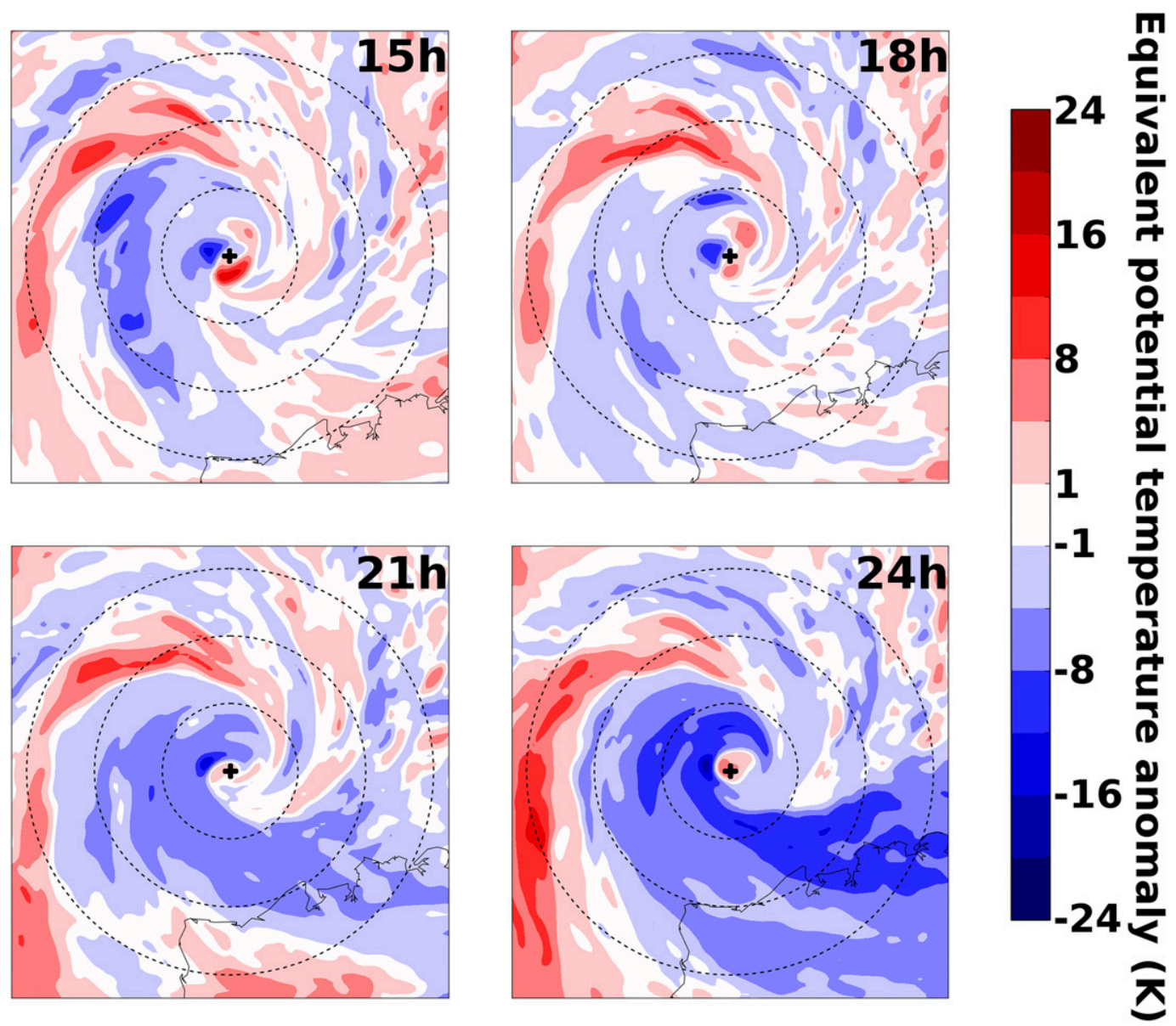

FIG. 8. Evolution of Hellen's 925-hPa $\theta_{e}$ anomaly from lead time $15 \mathrm{~h}$ (1500 UTC $30 \mathrm{Mar}$ ) to lead time $24 \mathrm{~h}$ (0000 UTC 31 Mar) with a 3-h time step in the AROME-IO experiment. The $\theta_{e}$ anomaly is computed with respect to lead time $9 \mathrm{~h}$ (0900 UTC $30 \mathrm{Mar})$ in a radial framework centered around the simulated TC center. Indicated are $\theta_{e}$ anomalies (K, shaded), the simulated TC center (black cross on each panel), and radii every $50 \mathrm{~km}$ from the center (dotted circles).

radius may be associated with downdrafts between rainbands. From $15 \mathrm{~h}$ onward, the downdrafts strengthen between the RMW and the $50-\mathrm{km}$ radius and gradually extend vertically in association with a decrease of $700-\mathrm{hPa}$ $\theta_{e}$ values. These downdrafts extending higher up are believed to be part of the transitory response of the TC secondary circulation to the environmental VWS constraint and to correspond to the gradual emergence of a stationary band complex (Willoughby et al. 1984). Eventually on the last four panels, the downdrafts cores are developing from the $300-400-\mathrm{hPa}$ level to the boundary layer as the response of the TC vortex becomes more and more balanced.

At $15 \mathrm{~h}$, some CTDAs also begin to appear at the top of the inflow layer $(850 \mathrm{hPa})$. At 6 hours later, the downdrafts are stronger and $\theta_{e}$ continue to decrease at the top of the inflow layer. Meanwhile, strong CTDAs are injecting this low-entropy air into the inflow layer. The same patterns are visible at $21 \mathrm{~h}$, albeit a bit weaker. Lead time $21 \mathrm{~h}$ corresponds to the onset of the rapid weakening in the model. The comparison between lead times 9 and $24 \mathrm{~h}$ reveal a clear change in the vertical distribution of $\theta_{e}$, similar to Fig. 9 in Riemer et al. (2010), with a tongue of depressed $\theta_{e}$ values coming down to the inflow layer close to the eyewall. This $\theta_{e}$ depression is located underneath the inner-core updrafts. At lead time $24 \mathrm{~h}$, the tip of this low $-\theta_{e}$ air tongue is even advected upward by the eyewall updrafts. Consistently with the idealized Carnot-cycle (Emanuel 1986), a weakening of the storm is observed. The core of downward velocities is stronger than in the beginning of the simulation, consistently with the more clearly established convective asymmetry on Fig. 7, that already suggested the gradual formation of a stationary band complex (Willoughby et al. 1984). The last two panels of Fig. 9 show that the tongue of low- $\theta_{e}$ air remains located just outside of the RMW (i.e., at the base 

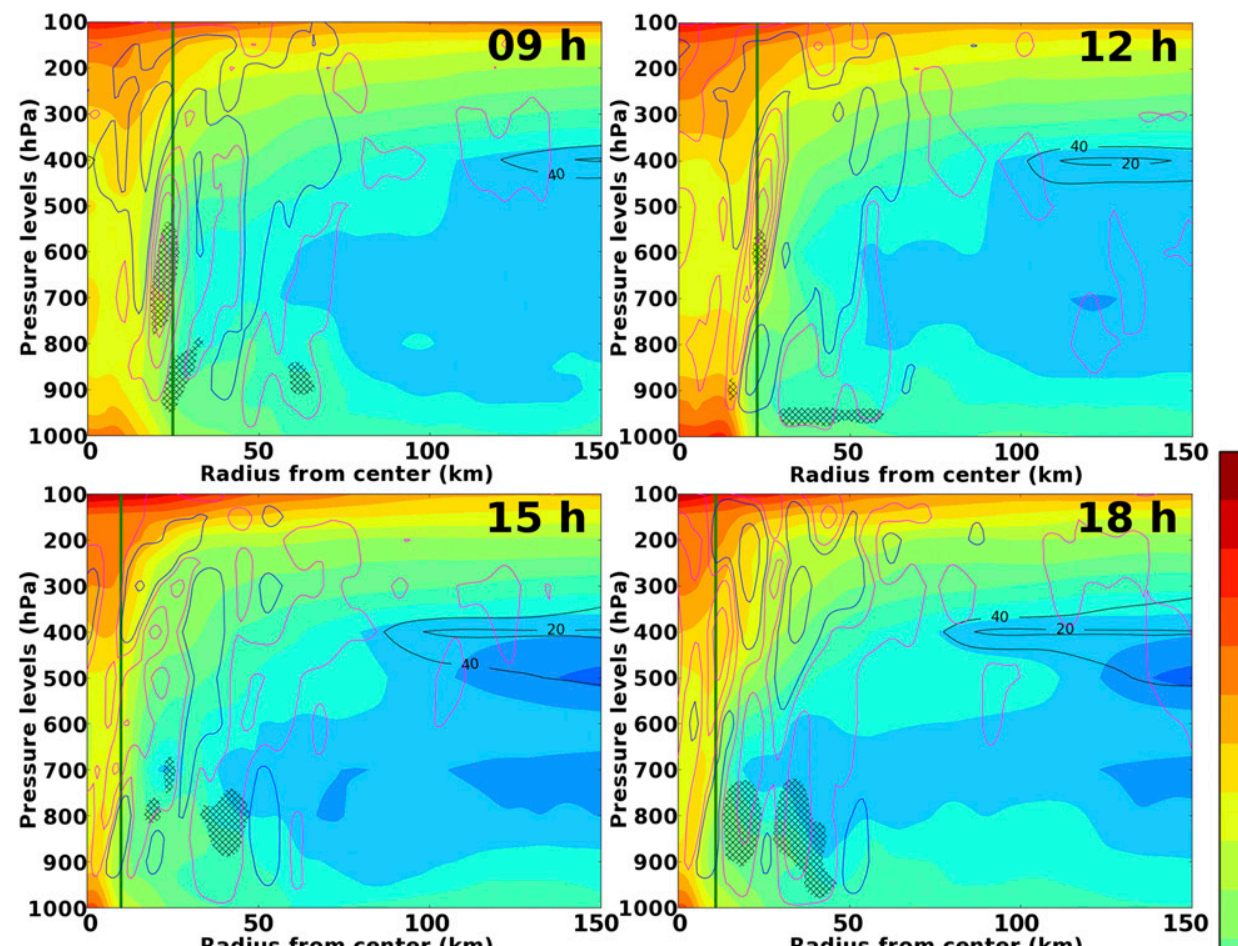

380
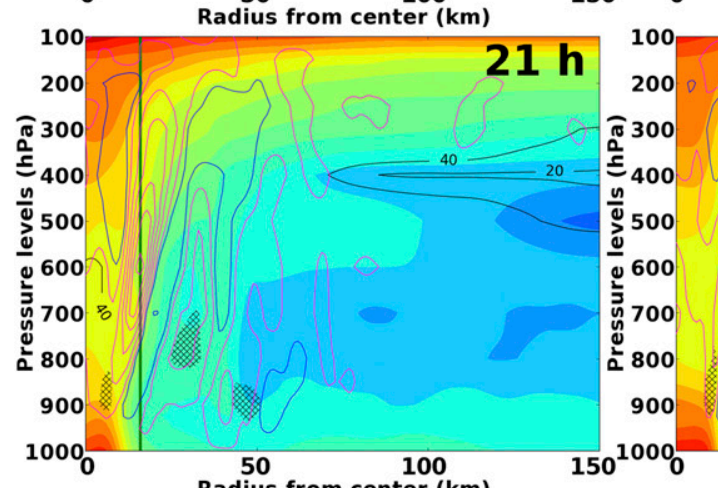

Radius from center $(\mathrm{km})$
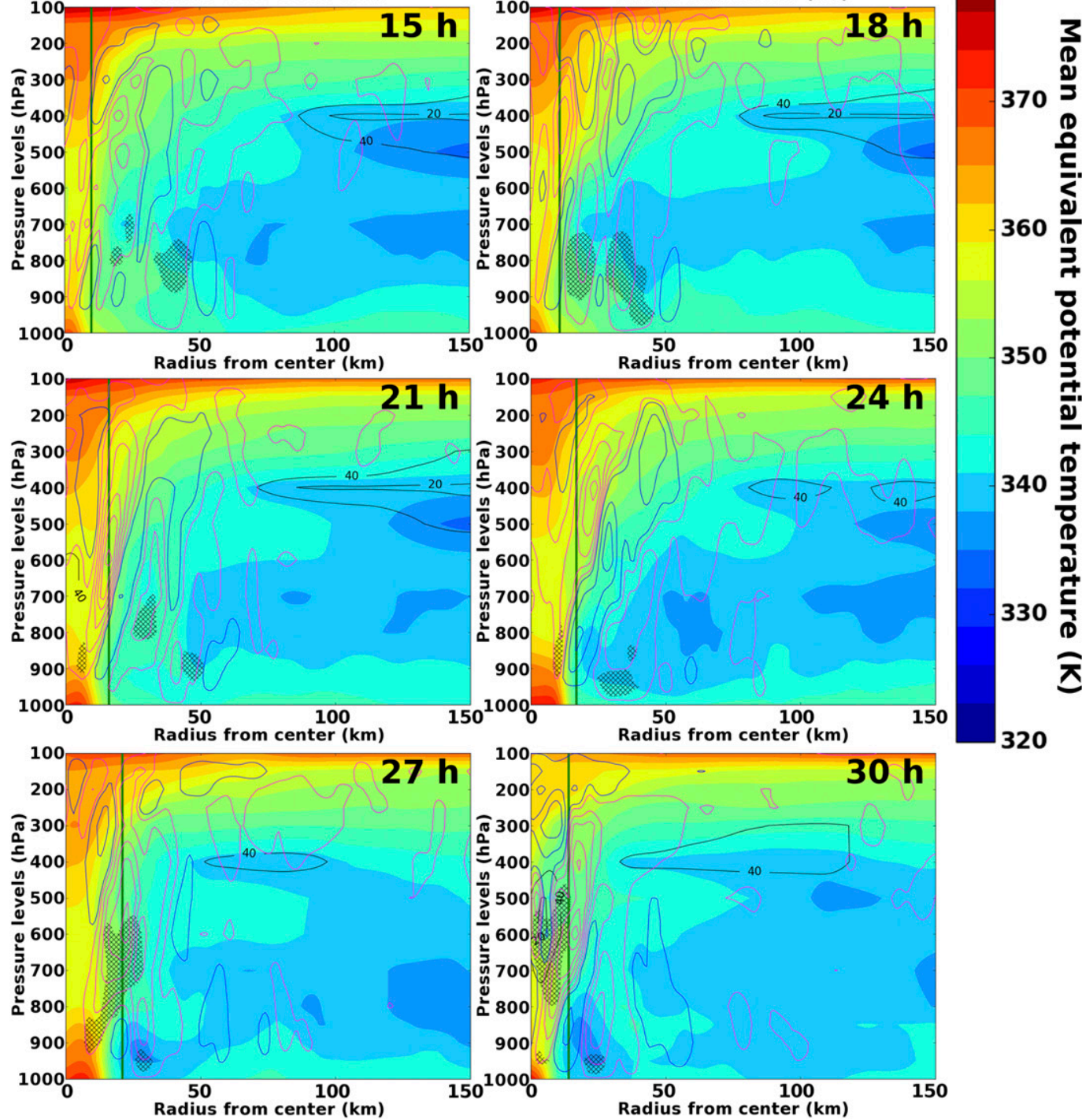

FIG. 9. Evolution of Hellen's northwestern quadrant from lead time $9 \mathrm{~h}$ (0900 UTC 30 Mar) to lead time $30 \mathrm{~h}$ (0600 UTC 31 Mar) with a 3-h time step in the AROME-IO experiment. All values are azimuthally averaged over the northwestern quadrant. Indicated are $\theta_{e}$ values $(\mathrm{K}$, shaded), downward vertical velocities (purple isolines from $0.1 \mathrm{~m} \mathrm{~s}^{-1}$ every $1 \mathrm{~m} \mathrm{~s}^{-1}$ ), upward vertical velocities (blue isolines from $0.5 \mathrm{~m} \mathrm{~s}^{-1}$ every $2 \mathrm{~m} \mathrm{~s}^{-1}$ ), and relative humidities lower than $40 \%$ (black isolines). The areas where the mean cooler $\theta_{e}$ downward advections are lower than $-5 \mathrm{~K} \mathrm{~h}^{-1}$ are hatched in black. The thick green line shows the location of the 925 -hPa radius of maximum winds. 
of the eyewall). The updrafts have been disrupted as only downdrafts are observed between the 20 and $50 \mathrm{~km}$ radii at lead time $27 \mathrm{~h}$. CTDAs are also injecting lower$\theta_{e}$ air directly into the eye. The 400 -hPa dry-air layer, delineated by the low $\theta_{e}$ and the $40 \%$ relative humidity isoline is as close as $50 \mathrm{~km}$ from the center. At lead time $30 \mathrm{~h}$, the same patterns are observed and the $\theta_{e}$ have significantly decreased within the RMW, especially above $500 \mathrm{hPa}$.

Although the 400-hPa dry-air layer appears to progress toward the TC core (Figs. 7, 9), this is not very clear if it plays any role in the onset of Hellen's rapid weakening through a direct interaction with the CTDAs in the inflow layer. Its presence may have favored downdrafts through the evaporation of precipitations. However, the $400-\mathrm{hPa}$ environmental wind and induced VWS constraint appear to be crucial in triggering the rapid weakening of the storm.

\section{d. Upper-level warm-core ventilation and $T C$ rapid weakening}

In this section, the focus is on the midlevel ventilation (Tang and Emanuel 2010) that represents the direct pathway in which the midlevel dry air presented in Figs. 2 and 6 could have directly affected the inner core of the TC.

A Hovmöller plot illustrates the progression of the 400-hPa dry air layer toward the TC center in the preferential source region of dry environmental air at this level (i.e., the northwestern quadrant of section 3a) (Fig. 10). The dry air located inside the RMW in the first time steps probably is probably a signature of the storm eye. Outside of the RMW, the strong gradient of relative humidity is collocated with a maximum of storm-relative radial inflow. From lead time $21 \mathrm{~h}$, the dry air progresses more quickly toward the TC center and eventually invades the RMW, as previously discussed in Fig. 9. This figure illustrates the direct influence of the 400-hPa dry layer on the TC core.

Following hydrostatic arguments, the strength of the surface low is directly linked to the warm-core intensity of the TC vortex. To follow the evolution of the simulated warm core, the temperature anomaly at the TC center is computed with respect to the initial profile averaged over the domain, to stay consistent with the definition of temperature anomalies in Zhang and Chen (2012). After the initial 6-h spinup, the model reproduces a typical TC warm core, with the strongest values located around $700 \mathrm{hPa}$, between 900 and $500 \mathrm{hPa}$ (Fig. 11a). A secondary warm anomaly can be seen between 200 and $400 \mathrm{hPa}$, associated to null to slightly downward vertical motion temporarily (Fig. 9). This warm anomaly is most likely caused by the subsiding air in the eye, which is a classic feature of mature TCs.

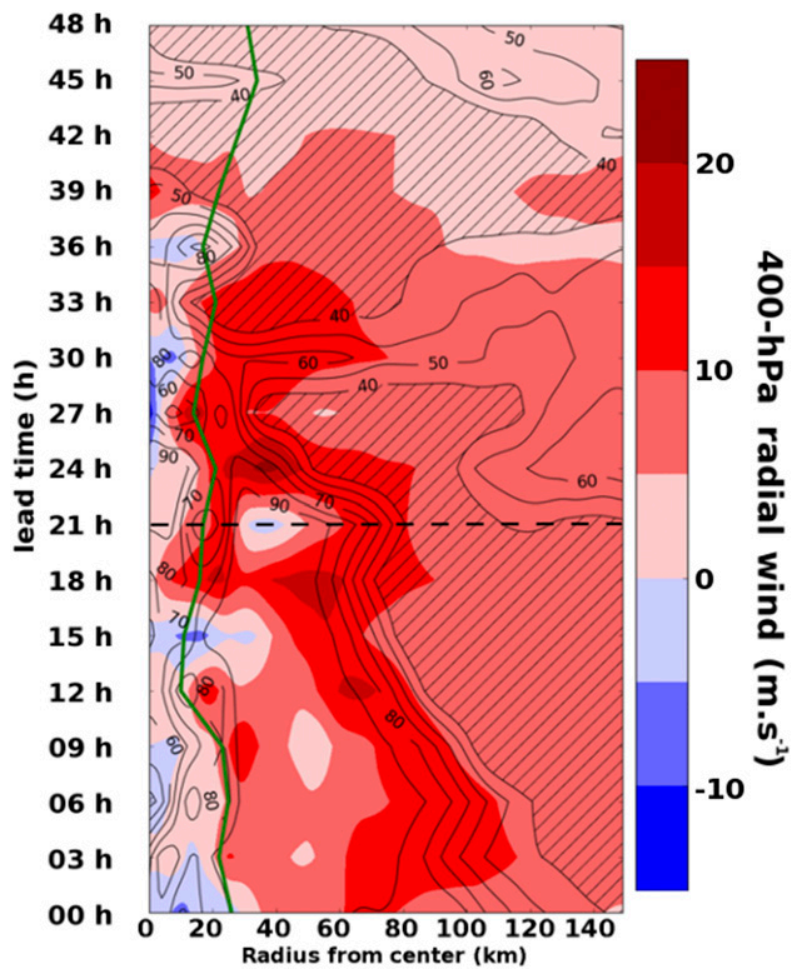

FIG. 10. Hovmöller plot of the simulated 400-hPa storm-relative radial wind $\left(\mathrm{m} \mathrm{s}^{-1}\right.$, shaded) and $400-\mathrm{hPa}$ relative humidity (\%, black contours; values lower than $40 \%$ are hatched). All values are azimuthally averaged over the northwestern quadrant. The thick green line shows the location of the $925-\mathrm{hPa}$ radius of maximum winds and the dashed black line indicates the time of TC Hellen's peak intensity. A positive radial wind corresponds to inflow.

At lead time $24 \mathrm{~h}$ (0000 UTC 31 March 2014), the temperature anomaly begins to decrease, especially in the upper levels (Fig. 11a). The timing is consistent with the arrival of midlevel dry air close to TC core (Fig. 6) and the development of low- $\theta_{e}$ values at the base of the eyewall (Fig. 9). These are the two pathways describe by Tang and Emanuel (2010) and Riemer et al. (2010) to weaken the warm core of a TC. In the following subsection, a discussion on the relative importance of each of these pathways is provided through the vortex spindown halftime theory. At 48-h lead time, the upperlevel warm core has vanished while the $700-\mathrm{hPa}$ warm core has substantially weakened (Fig. 11a).

The simulated central pressure is inversely correlated with the evolution of the mean intensity of the upper(400-200 hPa) and low-level (900-500 hPa) warm cores (Fig. 11b). After lead time $21 \mathrm{~h}$, however, as the central pressure rapidly increases, the upper-level temperature anomaly is better correlated with the surface pressure changes, which is confirmed by Pearson correlation coefficients (Fig. 11b). These results are consistent with those of Zhang and Chen (2012) who showed that the 

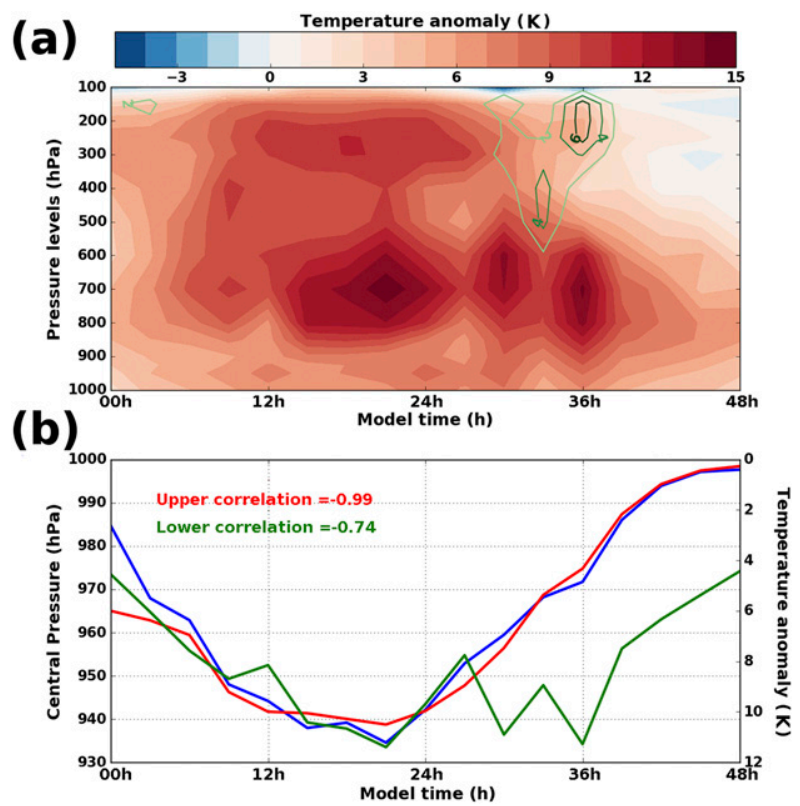

FIG. 11. The role of the warm-core structure changes in Hellen's rapid intensity changes. (a) Pressure-time plot of the temperature anomaly $\left(\mathrm{K}\right.$, shaded) and positive vertical velocities $\left(\mathrm{m} \mathrm{s}^{-1}\right.$, green contours) at the center of the simulated vortex. (b) Evolution of the upper (400-200 hPa, red line) and lower (900-500 hPa, green line) mean temperature anomalies $(\mathrm{K})$ computed relative to the initial mean profile in comparison with the simulated central pressure of the simulated TC (blue line, hPa). Numbers in the top-left corner of (b) show Pearson's correlation coefficients computed between either the upper or lower temperature anomaly and the central pressure for lead times $6-48 \mathrm{~h}$.

magnitude of the surface pressure drop caused by latent heat release increases with the height of the induced temperature anomaly. In other words, a warm anomaly is more efficient in deepening the surface low when it is located near $300 \mathrm{hPa}$ rather than $700 \mathrm{hPa}$. At 48-h lead time, the surface pressure is higher than that of the initial state, while the low-level temperature anomaly remains greater than $4 \mathrm{~K}$ and the upper-level anomaly is lower than $1 \mathrm{~K}$. The storm rapid collapse is therefore most likely primarily driven by the ventilation of the upperlevel warm core $(400-200 \mathrm{hPa}$ ) rather than by that of the stronger, but less influent, low-level (900-500 hPa) warm core. The rotational constraint also is lower at upper levels than at lower levels (Riemer and Montgomery 2011) (i.e., the radial advection is less deflected by azimuthal advection). The upper half of the warm core is thus easier to ventilate than its lower half.

\section{$e$. The relative influence of the low-versus midlevel processes in the rapid weakening through the vortex spindown theory}

The classic vortex spindown problem considers the evolution of an axisymmetric vortex above a rigid boundary normal to the axis of its rotation. The influence of friction at the surface of the boundary forces an ascending motion out of the inflow layer. Above the inflow layer, the parcels of fluid diverge and a radially outward motion is observed. Following the conservation of angular momentum, the particles spin more slowly as they move to larger radii and the tangential speed of the vortex gradually decreases. This simple concept can be used to understand some features of the TC secondary circulation (Willoughby 1979). In a mature TC, however, air parcels continue their ascent above the boundary layer instead of diverging outward, driven by positive buoyancy in the unstable atmosphere (Smith 2000). The vortex spindown theory thus explains, to a large extent, how a TC would weaken if all updrafts disappeared at once above the inflow layer (Montgomery et al. 2001). In the simulation, CTDAs and Madagascan horizontal advections are disrupting the VITC updrafts by lowering the $\theta_{e}$ in the inflow layer: the upward convective mass flux computed at $500 \mathrm{hPa}$ on a $40-\mathrm{km}$ disk is reduced by more than $40 \%$ in the first $10 \mathrm{~h}$ after the onset of rapid weakening, causing a partial spindown of the vortex.

Riemer et al. (2013) use an innovative tool to estimate the impact of a shutdown of the upward vertical mass flux on a TC vortex. Considering the half-time given by Eliassen and Lystad (1977), defined as the time required to reduce the initial tangential velocity by a factor of 2 , Riemer et al. (2013) found a time scale for the spindown of a TC without convective mass flux:

$$
t_{\text {half }}=\frac{H-h}{v \chi^{2} C_{D}},
$$

where $H$ is the fluid depth, $h$ is the height of the boundary layer, $v$ is the initial tangential velocity, $C_{D}$ is the wind speed-dependent drag coefficient, and $\chi$ is a reduction factor given by Eliassen and Lystad (1977) as the ratio of the tangential winds at surface and at the top of the boundary layer. To remain consistent with Riemer et al. (2013), we use the same estimated values for a TC vortex, $H-h=10^{4} \mathrm{~m}, \chi=0.8, C_{D}=3.8 \times 10^{-3}$ from Deacon's formula, and $v$ the maximum tangential speed at peak intensity.

In the simulated TC vortex, the maximum tangential winds is $v=62 \mathrm{~m} \mathrm{~s}^{-1}$. This yields a theoretical spindown half-time of $t_{\text {half }}=18.4 \mathrm{~h}$. However, it takes only $16-17 \mathrm{~h}$ for the maximum tangential wind of the simulated TC to effectively weaken by a factor 2 , suggesting a quicker weakening than if vortex spindown was the sole mechanism responsible for TC weakening. The spindown theory considers that all convective mass flux is stopped while the CTDAs cannot totally disrupt every vertical motion within the TC inner core, as indicated by the 
nonzero 500-hPa convective mass flux computed $10 \mathrm{~h}$ after the onset of TC weakening. The theoretical halftime thus only gives an upper bound of the influence of the CTDAs on the simulated TC weakening. Therefore, the warm-core erosion is not only coincidental and not a mere consequence of the spindown but is likely playing an instrumental role in Hellen's weakening. When the same reasoning is applied to the BT data, a theoretical spindown half-time of $17.8 \mathrm{~h}$ is found while the observed half-time is a little less than $12 \mathrm{~h}$. This difference suggests that for the real TC case also, a total spindown of the vortex is not able to fully explain the rapid weakening of VITC Hellen. The difference between the theoretical and observed half-times is larger when using the BT data compared to the AROME-IO data, possibly suggesting that in the real case scenario the erosion of the warm core might have played an even greater role than in the simulation or that other processes were at stake.

\section{Discussion}

As shown in Fig. 11a, Hellen's warm core is clearly separated in two distinctive halves at peak intensity. According to Kieu et al. (2016), this double warm-core structure has been observed in many very intense TC cases: the upper-level warm core is formed by a radial inflow located above the TC upper outflow, which advects warm air from the lower stratosphere thus lowering further the central sea level pressure. In the case of VITC Hellen, we have shown that the upper-level warm-core decay was closely linked to the rapid weakening of the storm. But its formation may also have played a dominant role in the rapid intensification of Hellen. However, the vertical resolution of the simulation above $200 \mathrm{hPa}$ is not sufficient to lead a meaningful investigation of the secondary circulation as in with Kieu et al. (2016).

Another specificity of this system is the location of the maximum VWS at midlevels (Fig. 6), similar to TC Dora (2007), although the VWS constraint was driven by the proximity of a deep upper to midlevel trough in the latter case (Leroux et al. 2013). This vertical structure of the VWS remains rather rare, as VWS is more often produced by jet streams driven by upper troughs leading to an increase of the VWS constraint with altitude. Despite a thorough analysis of the synoptic environment in the IFS model fields, no clear synoptic pattern explaining or driving this midlevel VWS has been elucidated. A trough can be depicted southwest of VITC Hellen at $400 \mathrm{hPa}$ and it likely fostered the southeastern upper-level outflow channel. However, this trough remains much too far to the south to have a potential direct impact on a VITC. Maybe the influence of this atmospheric structure was located or extended farther north than suggested by the global IFS model. The origin of the dry air layer was also investigated in the ERA-Interim reanalysis data through a basic retro-tracking method that only takes into account horizontal advective processes. Results showed that the air mass at $400 \mathrm{hPa}$ originated from the midlevel subtropical ridge, where drier conditions were produced due to synoptic subsidence.

In the BT, the TC center is located closer to the coastline than in the simulation from 1200 UTC 30 March (Fig. 3b) onward. Consequently, the Madagascan air mass may have penetrated the "dividing streamline" area earlier than in the experiment, triggering the storm decay $3 \mathrm{~h}$ earlier. Direct layer-wise advection may have thus been depressing the inflow-layer $\theta_{e}$ air earlier and with a greater amplitude than in the AROME-IO simulation. A sensitivity experiment with no landmass east of the system could be carried out to investigate the relative importance of the CTDAs versus that of the horizontal advection in the $\theta_{e}$ decrease. Additionally, the direct influence of higher land surface roughness on the pressure field, and thus on TC intensity, remains relatively marginal (Wong and Chan 2006). It is rather the very low moisture availability of the land surface that can cause a weakening of the TC by reducing the energy flux between the surface and the atmosphere. For a TC vortex with a $50-\mathrm{km}$ RMW spun up at $150 \mathrm{~km}$ from land, Wong and Chan (2006) experiments only showed a $10-\mathrm{hPa}$ weakening due to land proximity until landfall. In Hellen's case, as the simulated storm remains $30 \mathrm{~km}$ away from the Madagascan coast and has a 16$\mathrm{km}$ RMW, the contribution of land proximity to the rapid weakening is considered low. The last major TCs that have made landfall on the northwestern Madagascan coastline are Tropical Cyclone Fame during season 2007/08, intense Tropical Cyclone Bondo during season 2006/07, and intense Tropical Cyclones Kamisy and Andry during season 1983/84. None of those systems underwent a significant weakening when getting closer to the coastline, suggesting the Madagascan lower- $\theta_{e}$ air is not systematically affecting TC intensity.

In this case study, the environmental wind is computed on a $150-500-\mathrm{km}$ annulus. These radii have been chosen to remain consistent with the small size of the system. The $150-500-\mathrm{km}$ annulus has an inner radius larger than the simulated ROCI at peak intensity and an outer radius larger than three times this ROCI. In climatological studies e.g., Leroux et al. (2018), 200-800- or 200-1000-km annuli are used to quantify the environmental wind. The environmental wind computed using larger outer radii (e.g., 800 and $1000 \mathrm{~km}$ ) in our study shows weak environmental wind 
at $400 \mathrm{hPa}\left(3 \mathrm{~m} \mathrm{~s}^{-1}\right.$ at lead time $\left.21 \mathrm{~h}\right)$ and stronger values at $200 \mathrm{hPa}\left(5.5 \mathrm{~m} \mathrm{~s}^{-1}\right.$ at lead time $\left.21 \mathrm{~h}\right)$. The significant 400-hPa constraint is indeed located in the vicinity of the system (Fig. 6) and is important in advecting dry air close to the TC center at this level (Fig. 10). In fact, when considering a 100-200-km annulus that captures the area where the TC and the environmental wind meet, the averaged wind computed at peak intensity (lead time $21 \mathrm{~h}$ ) after subtracting the storm motion is $3.5 \mathrm{~m} \mathrm{~s}^{-1}$ at $200 \mathrm{hPa}$ and is maximum at $400 \mathrm{hPa}$ $\left(7.5 \mathrm{~m} \mathrm{~s}^{-1}\right)$, yielding a $400-850-\mathrm{hPa}$ VWS around $30 \mathrm{kt}$ $\left(15 \mathrm{~m} \mathrm{~s}^{-1}\right)$. This illustrates that the progression of the dry air at $400 \mathrm{hPa}$ toward the TC core is not impeded by the strong upper-level outflow, located at higher levels. For this reason, dry air is likely to progress more easily toward a strong TC core under a midlevel VWS constraint than under an upper-level VWS constraint. Additionally, the associated dry-air layer cannot be moistened by the sea at such levels and the rotational constraint is lower at upper levels than at lower levels (Riemer and Montgomery 2011). On the other hand, the radial gradient of $\theta_{e}$ is lower aloft than in the boundary layer (Fig. 9) and this may stunt the effect of midlevel ventilation compared to the CTSAs, as the lowest $\theta_{e}$ are located farther from the center than at lower levels. In the case of idealized experiments with a controlled environment, the wind fields remain homogeneous out of the TC direct influence but this is not the case in a real-case study. The strong heterogeneity of the 200- and 400-hPa winds in Fig. 6 also motivates the decision of using a smaller outer radii for wind averaging. The fact that the classic 200-800- and 200-1000-km annulii did not capture the $400-\mathrm{hPa}$ environmental wind maximum contributed to the difficulty of the operational intensity forecast.

The strongest downdrafts and CTDA patterns are seen at lead time $18 \mathrm{~h}$ on Fig. 9 but are also displayed earlier with weaker values. The low- $\theta_{e}$ air tongue reaches the base of the eyewall at lead time $21 \mathrm{~h}$ on Fig. 9. Meanwhile, the dry air layer reaches the vicinity of the eyewall only $6 \mathrm{~h}$ later (Fig. 10). The increase in central pressure begins at lead time $21 \mathrm{~h}$ and coincides with the beginning of the warm core decline (Fig. 3b). This suggests that the midlevel erosion only really begins once the weakening has been initiated by the CTDAs. The progression of the midlevel dry air accelerates from lead time $21 \mathrm{~h}$ (Fig. 10), suggesting that the CTDAs might facilitate the midlevel ventilation once they reach the base of the eyewall. Although the two pathways have been both thoroughly described (Tang and Emanuel 2010; Riemer et al. 2010; Onderlinde and Nolan 2017), little is known of their interactions and how they might facilitate each other.

\section{Conclusions and prospects}

The weakening of VITC Hellen over water is the greatest ever observed in the SWIO basin. This study investigated the mechanisms at play during this unprecedented rapid weakening, summarized in four schematic drawings (Fig. 12). Figure 12a illustrates the initial state of VITC Hellen as a mature TC without any significant environmental constraints. The TC secondary circulation is represented by black arrows: radial inflow in the boundary layer, updrafts in the eyewall and outflow aloft. The climatological $\theta_{e}$ minimum is shown in green, far from the TC inner core. When the environmental wind begins to strengthen at $400 \mathrm{hPa}$ (Fig. 12b), the TC response to the induced VWS is consistent with Riemer et al. (2010) with downdrafts forming upshear and the presence of low- $\theta_{e}$ air closer to the TC core around $700 \mathrm{hPa}$. To fully understand the role of environmental conditions, the analysis was more relevant at $400 \mathrm{hPa}$ than at upper levels (Fig. 6), while the common practice is to focus on the bulk VWS metric $(200-850 \mathrm{hPa})$. This partly explains why the rapid weakening was badly anticipated.

As the $400-\mathrm{hPa}$ environmental wind continues to strengthen, it begins to advect a very dry air layer toward the TC center (Fig. 12c). Meanwhile, downdrafts become more powerful as a result of the increase of VWS constraint on the TC vortex and the midlevel low$\theta_{e}$ air is injected into lower levels, and eventually reaches the inflow layer. These downward advections of low $-\theta_{e}$ air are found at the top of the inflow layer relatively close to the TC core within the upshear semicircle, consistently with the paradigm of Riemer et al. (2010) and Onderlinde and Nolan (2017). At this time, layer-wise advection from the Madagascan landmass could not directly reach the vicinity of the TC core as it was not located in the preferential source region of environmental air.

Eventually, the low $\theta_{e}$ air within the inflow layer is swept inward and comes under the eyewall updrafts. This tends to disrupt the vertical mass flux and probably facilitates the dry-air layer, advected by the VWS constraint, to penetrate into the TC core and erode the upper half of the warm core (Fig. 12d). Both the upperlevel warm-core erosion and the disruption of the updrafts participate in the weakening of the storm. The warm-core ventilation is indeed not only a mere consequence of vortex spindown triggered by the much lower $\theta_{e}$ air penetrating in the inflow layer. A possible retroactive interaction between the two pathways is 

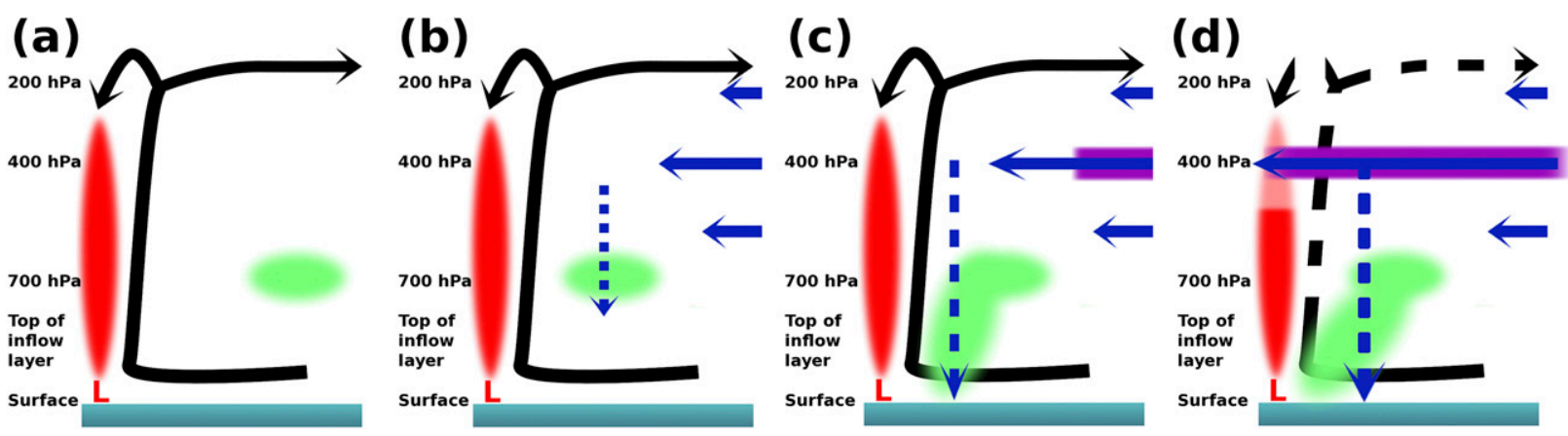

FIG. 12. Schematic drawing of the four stages leading to VITC Hellen's rapid weakening: (a) initial state, (b) increase of 400-hPa environmental wind shear, (c) response of the TC to wind shear and (d) warm core disruption. Black solid/dashed arrows represent the TC secondary active/disrupted circulation. Blue arrows represent the dynamic processes: the wind shear (solid) and induced downdrafts (dashed). The sea is represented by the blue area at the bottom. The magenta area represents the dry-air layer while the green area represents low $\theta_{e}$ values. The red area is representing the TC warm core with its associated surface low (L).

suggested as the CTDAs might facilitate midlevel ventilation, while the presence of dry air in the midlevels might fuel downdrafts through the evaporation of precipitations. Some future work in an idealized framework is needed to confirm and explore this hypothesis. Eventually, when the storm comes close enough to the Madagascan coast, low-entropy air originating from the landmass also participates in the general decrease of $\theta_{e}$ within the inflow layer. The $400-\mathrm{hPa}$ level with its environmental wind maximum and very dry air was therefore essential in the rapid weakening of VITC Hellen.

Note that as often in the SWIO basin, direct observations are lacking in the vicinity of TC's Hellen core to indubitably confirm the presence of midlevel dry air and the atypical 400-hPa VWS maximum.

For this complex TC case, the AROME-IO model showed some interesting skills and captured finescale mechanisms driving large intensity changes. Despite the small TC size, the representation of its structure and intensity proved to be consistent with the low-orbiting satellite infrared and microwave images. This gives credibility to AROME-IO to become a reliable tool for TC intensity prediction at RSMC La Réunion, especially during the warning time frame when model guidance is usually less skillful (DeMaria et al. 2014). Although classic environmental predictors were favorable for RI, the inner-core processes occurring during the RI phase were not addressed in this paper.

Last, the case of VITC Hellen has also highlighted the importance for TC forecasters to take into account the VWS in multiple layers, as suggested by Finocchio et al. (2016). Traditionally, the VWS is computed between the 200- and $850-\mathrm{hPa}$ levels and does not account for the VWS distribution within the midtroposphere. Some processes, like the ones described in this paper, are likely to remain undetected. From an operational point of view, it thus seems necessary to develop innovative products aimed at providing a quick and useful diagnosis of VWS constraints at all levels, using simple-to-read layouts, like hodographs for example. Such tools would help improve the TC forecaster analysis of model fields to better predict the impacts of VWS on TC intensity.

Acknowledgments. The authors acknowledge the contribution of David Barbary who set up the simulation and of P.Caroff. The reviews of an anonymous referee and of M.Riemer, who suggested in particular the use of the vortex spindown theory, greatly improved the paper.

\section{REFERENCES}

Braun, S. A., J. A. Sippel, and D. S. Nolan, 2012: The impact of dry midlevel air on hurricane intensity in idealized simulations with no mean flow. J. Atmos. Sci., 69, 236-257, https://doi.org/ 10.1175/JAS-D-10-05007.1.

Corbosiero, K. L., S. Abarca, and M. T. Montgomery, 2012: Vortex Rossby waves and secondary eyewall formation in a highresolution simulation of hurricane Katrina (2005). 30th Conf. on Hurricanes and Tropical Meteorology, Ponte Vedra Beach, FL, Amer. Meteor. Soc., 1A.6, https://ams.confex.com/ams/ 30Hurricane/webprogram/Paper205787.html.

Cram, T. A., J. Persing, M. T. Montgomery, and S. A. Braun, 2007: A Lagrangian trajectory view on transport and mixing processes between the eye, eyewall, and environment using a highresolution simulation of Hurricane Bonnie (1998). J. Atmos. Sci., 64, 1835-1856, https://doi.org/10.1175/JAS3921.1.

Dee, D., and Coauthors, 2011: The ERA-Interim reanalysis: Configuration and performance of the data assimilation system. Quart. J. Roy. Meteor. Soc., 137, 553-597, https://doi.org/ 10.1002/qj.828.

DeMaria, M., and J. Kaplan, 1994: A Statistical Hurricane Intensity Prediction Scheme (SHIPS) for the Atlantic basin. Wea. Forecasting, 9, 209-220, https://doi.org/10.1175/15200434(1994)009<0209:ASHIPS>2.0.CO;2. 
—, M. Mainelli, L. K. Shay, J. A. Knaff, and J. Kaplan, 2005: Further improvements to the Statistical Hurricane Intensity Prediction Scheme (SHIPS). Wea. Forecasting, 20, 531-543, https://doi.org/10.1175/WAF862.1.

— C. R. Sampson, J. A. Knaff, and K. D. Musgrave, 2014: Is tropical cyclone intensity guidance improving? Bull. Amer. Meteor. Soc., 95, 387-398, https://doi.org/10.1175/BAMS-D12-00240.1.

Dvorak, V. F., 1975: Tropical cyclone intensity analysis and forecasting from satellite imagery. Mon. Wea. Rev., 103, 420-430, https://doi.org/10.1175/1520-0493(1975)103<0420: TCIAAF $>2.0 . \mathrm{CO} ; 2$.

, 1984: Tropical cyclone intensity analysis using satellite data. NOAA Tech. Rep. 11, 45 pp.

Eliassen, A., and M. Lystad, 1977: The Ekman layer of a circular vortexa numerical and theoretical study. Geophys. Norv., 31, 1-16.

Emanuel, K. A., 1986: An air-sea interaction theory for tropical cyclones. Part I: Steady-state maintenance. J. Atmos. Sci., 43, 585-604, https://doi.org/10.1175/1520-0469(1986)043<0585: AASITF $>2.0 . \mathrm{CO} ; 2$.

Faure, G., P. Chambon, and C. Lebeaupin-Brossier, 2018: MétéoFrance new NWP models in tropical regions. 33rd Conf. on Hurricanes and Tropical Meteorology, Ponte Vedra Beach, FL, Amer. Meteor. Soc., 8B.2, https://ams.confex.com/ams/ 33HURRICANE/webprogram/Paper339675.html.

Figa-Saldaña, J., J. J. Wilson, E. Attema, R. Gelsthorpe, M. Drinkwater, and A. Stoffelen, 2002: The Advanced Scatterometer (ASCAT) on the Meteorological operational (MetOp) platform: A follow on for European wind scatterometers. Can. J. Rem. Sens., 28, 404-412, https://doi.org/ $10.5589 / \mathrm{m} 02-035$.

Finocchio, P. M., and S. J. Majumdar, 2017: A statistical perspective on wind profiles and vertical wind shear in tropical cyclone environments of the Northern Hemisphere. Mon. Wea. Rev., 145, 361-378, https://doi.org/10.1175/MWR-D-16-0221.1.

,$- \ldots$, D. S. Nolan, and M. Iskandarani, 2016: Idealized tropical cyclone responses to the height and depth of environmental vertical wind shear. Mon. Wea. Rev., 144, 2155-2175, https:// doi.org/10.1175/MWR-D-15-0320.1.

Frank, W., and E. Ritchie, 2001: Effects of vertical wind shear on the intensity and structure of numerically simulated hurricanes. Mon. Wea. Rev., 129, 2249-2269, https://doi.org/10.1175/15200493(2001)129<2249:EOVWSO>2.0.CO;2.

Frederick, W. J., 2003: The rapid intensification and subsequent rapid weakening of Hurricane Lili as compared with historical hurricanes. Wea. Forecasting, 18, 1295-1298, https://doi.org/ 10.1175/1520-0434(2003)018<1295:TRIASR $>2.0$. CO;2.

Hendricks, E. A., M. T. Montgomery, and C. A. Davis, 2004: The role of vortical hot towers in the formation of Tropical Cyclone Diana (1984). J. Atmos. Sci., 61, 1209-1232, https://doi.org/ 10.1175/1520-0469(2004)061<1209:TROVHT>2.0.CO;2.

Kaplan, J., and M. DeMaria, 2003: Large-scale characteristics of rapidly intensifying tropical cyclones in the North Atlantic basin. Wea. Forecasting, 18, 1093-1108, https://doi.org/10.1175/ 1520-0434(2003)018<1093:LCORIT>2.0.CO;2.

,-- , and J. A. Knaff, 2010: A revised tropical cyclone rapid intensification index for the Atlantic and eastern North Pacific basins. Wea. Forecasting, 25, 220-241, https://doi.org/10.1175/ 2009WAF2222280.1.

Kieu, C., V. Tallapragada, D.-L. Zhang, and Z. Moon, 2016: On the development of double warm-core structures in intense tropical cyclones. J. Atmos. Sci., 73, 4487-4506, https://doi.org/ 10.1175/JAS-D-16-0015.1.
Knaff, J. A., D. P. Brown, J. Courtney, G. M. Gallina, and J. L. Beven, 2010: An evaluation of Dvorak technique-based tropical cyclone intensity estimates. Wea. Forecasting, 25, 1362-1379, https://doi.org/10.1175/2010WAF2222375.1.

Kummerow, C., W. Barnes, T. Kozu, J. Shiue, and J. Simpson, 1998: The Tropical Rainfall Measuring Mission (TRMM) sensor package. J. Atmos. Oceanic Technol., 15, 809-817, https://doi.org/ 10.1175/1520-0426(1998)015<0809:TTRMMT>2.0.CO;2.

Leroux, M.-D., M. Plu, D. Barbary, F. Roux, and P. Arbogast, 2013: Dynamical and physical processes leading to tropical cyclone intensification under upper-level trough forcing. J. Atmos. Sci., 70, 2547-2565, https://doi.org/10.1175/JAS-D-12-0293.1.

,-- , and F. Roux, 2016: On the sensitivity of tropical cyclone intensification under upper-level trough forcing. Mon. Wea. Rev., 144, 1179-1202, https://doi.org/10.1175/MWR-D15-0224.1.

— J. Meister, D. Mekies, A.-L. Dorla, and P. Caroff, 2018: A climatology of southwest Indian Ocean tropical systems: Their number, tracks, impacts, sizes, empirical maximum potential intensity, and intensity changes. J. Appl. Meteor. Climatol., 57, 1021-1041, https://doi.org/10.1175/JAMC-D-17-0094.1.

Madec, G., 2008: NEMO ocean engine. Note du Pôle de modélisation de 1'Institut Pierre-Simon Laplace, Tech. Rep. 27, 396 pp, https://doi.org/10.5281/zenodo.1464816.

Molinari, J., J. Frank, and D. Vollaro, 2013: Convective bursts, downdraft cooling, and boundary layer recovery in a sheared tropical storm. Mon. Wea. Rev., 141, 1048-1060, https:// doi.org/10.1175/MWR-D-12-00135.1.

Montgomery, M. T., and R. J. Kallenbach, 1997: A theory of vortex Rossby waves and its application to spiral bands and intensity changes in hurricanes. Quart. J. Roy. Meteor. Soc., 123, 435-465, https://doi.org/10.1002/qj.49712353810.

_ H. D. Snell, and Z. Yang, 2001: Axisymmetric spindown dynamics of hurricane-like vortices. J. Atmos. Sci., 58, 421-435, https:// doi.org/10.1175/1520-0469(2001)058<0421:ASDOHL>2.0.CO;2.

— M. E. Nicholls, T. A. Cram, and A. B. Saunders, 2006: A vortical hot tower route to tropical cyclogenesis. J. Atmos. Sci. 63, 355-386, https://doi.org/10.1175/JAS3604.1.

Onderlinde, M. J., and D. S. Nolan, 2017: The tropical cyclone response to changing wind shear using the method of time-varying point-downscaling. J. Adv. Model. Earth Syst., 9, 908-931, https://doi.org/10.1002/2016MS000796.

Pergaud, J., V. Masson, S. Malardel, and F. Couvreux, 2009: A parameterization of dry thermals and shallow cumuli for mesoscale numerical weather prediction. Bound.-Layer Meteor. 132, 83, https://doi.org/10.1007/s10546-009-9388-0.

Pinty, J.-P., and P. Jabouille, 1998: A mixed-phased cloud parameterization for use in a mesoscale non-hydrostatic model: Simulations of a squall line and of orographic precipitation. Conf. on Cloud Physics: 14th Conf. on Planned and Inadvertent Weather Modification, Everett, WA, Amer. Meteor. Soc., 6B.4, http://195.83.22.22/mesonh/dir_publication/pinty_ jabouille_ams_ccp1998.pdf.

Riemer, M., and M. T. Montgomery, 2011: Simple kinematic models for the environmental interaction of tropical cyclones in vertical wind shear. Atmos. Chem. Phys., 11, 9395-9414, https://doi.org/10.5194/acp-11-9395-2011.

$\longrightarrow, \ldots$, and M. E. Nicholls, 2010: A new paradigm for intensity modification of tropical cyclones: Thermodynamic impact of vertical wind shear on the inflow layer. Atmos. Chem. Phys., 10, 3163-3188, https://doi.org/10.5194/acp-10-3163-2010.

,-- , and -2013 : Further examination of the thermodynamic modification of the inflow layer of tropical cyclones 
by vertical wind shear. Atmos. Chem. Phys., 13, 327-346, https://doi.org/10.5194/acp-13-327-2013.

Seity, Y., P. Brousseau, S. Malardel, G. Hello, P. Bénard, F. Bouttier, C. Lac, and V. Masson, 2011: The AROME-France convectivescale operational model. Mon. Wea. Rev., 139, 976-991, https:// doi.org/10.1175/2010MWR3425.1.

Sitkowski, M., J. P. Kossin, and C. M. Rozoff, 2011: Intensity and structure changes during hurricane eyewall replacement cycles. Mon. Wea. Rev., 139, 3829-3847, https://doi.org/10.1175/ MWR-D-11-00034.1.

Smith, R. K., 2000: The role of cumulus convection in hurricanes and its representation in hurricane models. Rev. Geophys., 38 465-489, https://doi.org/10.1029/1999RG000080.

Tang, B., and K. Emanuel, 2010: Midlevel ventilation's constraint on tropical cyclone intensity. J. Atmos. Sci., 67, 1817-1830, https://doi.org/10.1175/2010JAS3318.1.

Velden, C., and Coauthors, 2006: The Dvorak tropical cyclone intensity estimation technique: A satellite-based method that has endured for over 30 years. Bull. Amer. Meteor. Soc., 87, 1195-1210, https://doi.org/10.1175/BAMS-87-91195.

Wang, Y., 2002: Vortex Rossby waves in a numerically simulated tropical cyclone. Part II: The role in tropical cyclone structure and intensity changes. J. Atmos. Sci., 59, 1239-1262, https://doi.org/ 10.1175/1520-0469(2002)059<1239:VRWIAN>2.0.CO;2.

Willoughby, H., 1979: Forced secondary circulations in hurricanes. J. Geophys. Res., 84, 3173-3183, https://doi.org/10.1029/ JC084iC06p03173.

Willoughby, H. E., F. D. Marks Jr., and R. J. Feinberg, 1984: Stationary and moving convective bands in hurricanes. J. Atmos. Sci., 41, 3189-3211, https://doi.org/10.1175/1520-0469(1984) $041<3189:$ SAMCBI $>2.0 . \mathrm{CO} ; 2$.

Wong, M. L., and J. C. Chan, 2006: Tropical cyclone motion in response to land surface friction. J. Atmos. Sci., 63, 1324-1337, https://doi.org/10.1175/JAS3683.1.

— and — 2007: Modeling the effects of land-sea roughness contrast on tropical cyclone winds. J. Atmos. Sci., 64, 32493264, https://doi.org/10.1175/JAS4027.1.

Zhang, D.-L., and H. Chen, 2012: Importance of the upper-level warm core in the rapid intensification of a tropical cyclone. Geophys. Res. Lett., 39, L02806, https://doi.org/10.1029/ 2011 GL050578.

Zhang, X., Q. Xiao, and P. J. Fitzpatrick, 2007: The impact of multisatellite data on the initialization and simulation of Hurricane Lili's (2002) rapid weakening phase. Mon. Wea. Rev., 135, 526-548, https://doi.org/10.1175/MWR3287.1. 